\title{
Picolinic acid, a catabolite of tryptophan, has an anabolic effect on bone in vivo
}

\author{
Gustavo Duque, MD, Ph.D. ${ }^{1,2}$, Christopher Vidal, Ph.D. ${ }^{3}$, Wei Li, M.Sc. ${ }^{3}$, \\ Ahmed Al Saedi, Ph.D. ${ }^{1,2}$, Mamdouh Khalil, Ph.D. ${ }^{4}$, Chai K. Lim, Ph.D. ${ }^{5}$ \\ Damian E. Myers, Ph.D. ${ }^{1,2}$, Gilles J. Guillemin, Ph.D. ${ }^{5}$
}

\begin{abstract}
${ }^{1}$ Australian Institute for Musculoskeletal Science (AIMSS), The University of Melbourne and Western Health, St Albans, VIC, 3021, Australia

${ }^{2}$ Department of Medicine-Western Health, Melbourne Medical School, The University of Melbourne, St Albans, VIC, 3021, Australia

${ }^{3}$ Sydney Medical School Nepean, The University of Sydney, Penrith, NSW, Australia

${ }^{4}$ ANZAC Research Institute, Sydney Medical School Concord, The University of Sydney, Concord, NSW, Australia

${ }^{5}$ Neuroinflammation Group, Faculty of Medicine and Health Sciences, Macquarie University, Sydney, NSW, Australia
\end{abstract}

Correspondence to: Prof. Gustavo Duque, MD, Ph.D., FRACP, FGSA

Australian Institute for Musculoskeletal Science (AIMSS)

The University of Melbourne and Western Health

176 Furlong Road, St. Albans, VIC 3021, Australia

Tel: +61 383958121

Email: gustavo.duque@unimelb.edu.au

This is the author manuscript accepted for publication and has undergone full peer review but has not been through the copyediting, typesetting, pagination and proofreading process, which may lead to differences between this version and the Version of Record. Please cite this article as doi: $10.1002 / \mathrm{jbmr} .4125$

This article is protected by copyright. All rights reserved. 
Keywords: Osteoporosis; osteoanabolics; kynurenine; picolinic acid; osteoblasts; Wnts.

\begin{abstract}
Fractures due to osteoporosis have a severe impact on our older population. Reports of side effects with commonly prescribed osteoporosis drugs have led to the investigation of new and safer treatments with novel mechanisms of action. Picolinic acid (PIC), a catabolite of tryptophan, induces in vitro osteogenic differentiation of mesenchymal stem cells. Here we demonstrate that PIC has an anabolic effect on bone in vivo by increasing bone formation, bone mass, and bone strength in normal and ovariectomized C57BL/6 mice. Activation of the osteogenic pathways triggered this osteoanabolic response without any cross-related effects on mineral absorption or calciotropic hormones. As PIC was also well tolerated and absorbed with no side effects, it is an ideal potential candidate for the treatment for osteoporosis.
\end{abstract}

This article is protected by copyright. All rights reserved. 


\section{Introduction}

Osteoporosis causes painful fractures that lead to decreased mobility, reduced health generally, and significantly reduced quality of life due to chronic pain, disability, and the inability to perform daily activities independently. ${ }^{(1)}$ In addition, some $25 \%$ of patients with hip fractures are eventually admitted to residential aged-care facilities, with a major cost to the health system. ${ }^{(1)}$ People with osteoporosis can also die prematurely, especially if they sustain hip or spinal fractures. ${ }^{(2)}$

Antiresorptive therapies have a limited capacity to increase bone mineral density (BMD). They decrease the risk of non-vertebral fractures by just 20\%. ${ }^{(3)}$ Currently, the alternative treatment approach using osteoanabolic (bone-forming) drugs is limited to three drugs (teriparatide, abaloparatide and romosozumab). Teriparatide and abaloparatide have a similar mechanism of action and can be administered for a maximum of only 24 months. ${ }^{(3)}$ Further, new treatments such as the anabolic romosozumab and the anti-resorptive odanacatib, have shown promising results. However, they have been associated with unexpected cardiovascular effects, which have affected or delayed their approval process. ${ }^{(4-6)}$ Antiresorptive and osteoanabolic drugs currently used have

This article is protected by copyright. All rights reserved. 
limitations. This is due to their adverse effects or rare, but serious, complications over time (i.e., for antiresorptives, femoral fractures that are atypical and osteonecrosis of the jaw; and for anabolics, osteosarcoma). ${ }^{(4)}$ As a result, investigations and tests for new therapeutic targets have been conducted. These have particularly targeted therapies with an anabolic effect that allow more tolerance to extensive utilization, and avoid the side effects associated with extended use of traditional drugs.

Amongst those novel regulators of bone metabolism, the essential amino acid tryptophan, which serves several vital purposes such as nitrogen balance in adults and growth in infants, is also associated with the regulation of bone metabolism. ${ }^{(7)}$ Only about 3\% of the tryptophan obtained from our diet is converted into nicotinamide adenine dinucleotide (NAD+). This conversion happens in the liver and can help prevent the symptoms associated with niacin (Vitamin B3) deficiency, especially in conditions of low dietary intake of this vitamin. Tryptophan is also converted to serotonin, which is a neurotransmitter generated in the brain and the gut through the activation of the enzyme tryptophan hydroxylase 1 (TPH-1) that regulates appetite, gut motility, and mood. Bone formation is inhibited by gut-derived serotonin (GDS) due to its effect on osteoblast (bone-forming cell) differentiation, ${ }^{(8,9)}$ therefore, inhibition of GDS has been proposed as a new strategy for the treatment of osteoporosis. ${ }^{(10-12)}$ However, there is some controversy surrounding this hypothesis - research teams have produced contradictory results, and the side effects of blocking GDS production in humans are potentially severe. ${ }^{(13)}$

While several groups have studied the functions of serotonin in bone metabolism, the involvement of the alternative tryptophan catabolic pathway (the kynurenine [KYN] pathway) (Figure 1) in bone metabolism remains poorly understood; even though 95\% of dietary tryptophan is metabolized by 
this pathway. The process starts with the activation of the enzyme indoleamine 2,3-dioxygenase-1 (IDO-1). Tryptophan is catabolized into KYN, which is subsequently converted into either quinolinic acid or picolinic acid (PIC). These two compounds have been linked with regulation of oxidative stress and cell differentiation. ${ }^{(14-17)}$

We have previously reported that, under osteogenic conditions, human mesenchymal stem cells (MSCs) have higher levels ( 50-fold) of IDO-1 activity as compared with MSCs treated with growth media alone without showing changes in TPH-1 activity. ${ }^{(18)}$ This result was associated with a higher production of PIC without affecting serotonin levels in the supernatants of MSCs undergoing osteoblastogenesis. In addition, the complete bone phenotype in four-month-old female Ido1-/- mice displayed severe osteopenia, which was associated with poor bone formation. This was independent of the levels of serotonin production within the brain and gut. ${ }^{(18)}$ Furthermore, we characterized the anabolic effect of PIC on bone marrow stromal cells (BMSC) isolated from Ido1 ${ }^{-/-}$ mice and human MSCs grown for seven days in osteogenic media that contained PIC. Treatment with PIC fully recovered the osteogenic capacity of the Ido1 ${ }^{-/}$BMSC and induced higher levels of osteoblastogenesis in the human MSCs; this effect was stronger than quinolinic acid. ${ }^{(18)}$

The role of the KYN pathway has also been previously reported in human studies. Apalset et al. found there was a correlation between the serum levels of interferon gamma (IFN $\gamma$ )-induced kynurenines and the BMD of middle-aged (46-49 years) and older (71-74 years) participants ( $\mathrm{n}=$ 5312). ${ }^{(19)}$ Their study showed the association of BMD with serum concentrations of two IFN $\gamma$ mediated kynurenines: xanthurenic acid and 3-hydroxyanthranilic acid. Another study by Forrest et al. ${ }^{(20)}$ examined markers of oxidative stress and concentrations of KYN pathway metabolites in the plasma of osteoporosis patients before and after two years of treatment with either etidronate or 
raloxifene. The authors showed that in osteoporosis, the tryptophan metabolism is altered to such a point that it is very likely to contribute to the progression of the disease. Their conclusion was that: "the metabolism of tryptophan through the KYN pathway could represent a novel target for the development of new drugs for the treatment of osteoporosis.”

Given this substantial body of evidence suggesting a strong anabolic effect of PIC on osteogenesis, in this study, we hypothesized that PIC acts as a new, safe, and effective anabolic treatment for osteoporosis in vivo. We tested this hypothesis by assessing on the effect of oral PIC on the bone of OVX C57BL/6 mice - an accepted osteoporosis murine model - along with the respective Shamoperated controls.

\section{Materials and Methods}

Animals. Nine-month-old oophorectomized (OVX) and Sham-operated C57BL/6 mice were purchased from the Animal Resources Centre in Perth, WA. After 2 weeks of acclimation, we divided the animals into eight experimental sub-groups of mice ( $n=10$ per group): 1 ) vehicle-treated Sham; 2) Sham PIC low dose (0.25 g/kg/d); 3) Sham PIC mid dose (0.5 g/kg/d); 4) Sham PIC high dose (1 g/kg/d); 5) vehicle-treated OVX; 6) OVX PIC low dose (0.25 g/kg/d); 7) OVX PIC mid dose (0.5 g/kg/d); 8) OVX PIC high dose (1 g/kg/d). Doses were calculated based in vitro data ${ }^{(18)}$ compared with preliminary in vivo data, ${ }^{(21,22)}$ and analyzed using in vitro-in vivo correlation (IVIVC) Toolkit (Pharsight, St. Louis, MO, USA). Briefly, the known pharmacokinetics of PIC in vivo $^{(22)}$ were analyzed via a pseudomono-compartmental model and deconvolution of PIC plasma concentration profiles. The calculated in vivo absorption fractions were correlated with the in vitro PIC dissolution data previously obtained by our team ${ }^{(18)}$ with a linear IVIVC being obtained for 
PIC. We used mice that were treated with water as our controls. Treatment was added to the animal drinking water for an overall time of 6 weeks. They were housed in cages in a room with limited access and monitored weekly for behavior and activity, weight, water, and food (regular chow diet) consumption. Animal husbandry adhered to Sydney Local Health District Animal Welfare Committee. Investigators were blinded during allocation, animal handling, and endpoint measurements.

Reagents, Antibodies and Media. All reagents for cell culture, PIC and analytical grade reagents were obtained from Sigma (Sigma-Aldrich, Sydney, NSW, Australia) unless otherwise specified. Antibodies for western blotting were purchased from Santa Cruz Biotechnology (Santa Cruz, CA).

Gas Chromatography-Mass Spectrometry (GC-MS). For absorption analysis of minerals in the duodenum, tissue was dissected and left to dry up in room temperature. $100 \mathrm{mg}$ of tissue were dissolved in nitric acid and concentrations of nine minerals were quantified in the tissue homogenates using an Agilent 5973 mass selective detector (Agilent, Santa Clara, CA, USA) equipped with an Agilent Technologies 7683 auto-sampler. The mean intra- and inter-assay coefficient of the variation (\%CV) of those minerals was 8.6\% (within the acceptable range of 6$9 \%)$.

For absorption analysis of PIC and serum levels of minerals, the mice were euthanized after 6 weeks of the treatment. Cardiac puncture was performed to collect blood. Serum of PIC- and vehicle-treated animals was obtained by centrifugation. Quantification of PIC in serum samples was conducted as described above. ${ }^{(18)}$ Briefly, serum samples were deproteinized with $5 \%$ (final concentration, $\mathrm{w}+\mathrm{v}$ ) trichloroacetic acid and derivatized with trifluoracetic acid and hexafluoro isopropanol to form the hexafluoro isopropyl ester. The esterified product was reconstituted in 
toluene and $1 \mu$ l of the mixture was injected into an Agilent 7890 GC, interfaced to an Agilent 5975 single quadrupole mass selective detector equipped with an Agilent Technologies 7683 autosampler operating at negative ionization mode. The Agilent ChemStation software (Agilent, Santa Clara, CA, USA) was used to run the system. The intra- and inter-assay CV of PIC was within the acceptable range of $7-10 \%$.

Biochemical Analysis. The concentration of calciotropic hormones was quantified using ELISA kits for parathyroid hormone (PTH) (Immunotopics Inc San Clemente, CA, USA) and 25 hydroxyvitamin D (25(OH)D) (ImmunoDiagnostic Systems Ltd, UK).

$\boldsymbol{\mu}$-CT Analysis. Following the removal of soft tissues and fixation overnight in $4 \%$ paraformaldehyde, $\mu$-CT was performed on the left femur and lumbar vertebrae. The distal metaphysis of the femur and the body of the vertebrae were scanned using a Skyscan 1072 microCT instrument (Skyscan, Antwerp, Belgium). Distal femurs were imaged at a nominal isotropic voxel resolution of $7 \mu \mathrm{m}$. An approximate length of $5 \mathrm{~mm}$ was scanned starting from the distal-most aspect of the condyles and progressing proximally to include the entire metaphysis and some diaphysis. Image acquisition was done at $100 \mathrm{kV}$ and $98 \mu \mathrm{A}$, with a $0.9^{\circ}$ rotation between frames. The three-dimensional reconstructions were generated using the two-dimensional images. Images were analyzed with the 3D Creator software supplied with the equipment to obtain quantitative data. We used the recommendations of the American Society of Bone and Mineral Research for the nomenclature and abbreviations of 3D- $\mu \mathrm{CT}$ parameters. ${ }^{(23)}$

Three-Point Bending. The bones were soaked in a PBS for $24 \mathrm{~h}$ prior to the experiment. We placed the tibia in a specially designed holder that was fixed to the ends of the bone and then deployed a measured amount of force perpendicular to the midpoint of the anterior side of the tibial diaphysis. The force was applied (at a rate of $0.498 \mathrm{~mm} \cdot \mathrm{s}-1$ ) utilizing a flat-tipped wedge, which 
was connected to a motorized force transducer (World Precision Instruments, FL, USA), controlled by a Hayden Kerk IDEA Drive Interface program. A trans bridge amplifier (World Precision Instruments) was used to amplify force and this was recorded with WinDaq data acquisition software (DATAQ Instruments, OH, USA).

Histological and Histomorphometrical Analysis of Bone. Intraperitoneal injections of demeclocycline (20 mg/kg) (Sigma Chemicals, St. Louis, MO, USA) were performed on the treated groups at days 5 and 2 before sacrifice to measure tetracycline labeling for the assessment of dynamic histomorphometry.

After euthanasia, one side femur from each animal in each group was removed. Tissue was then fixed in 70\% ethanol, dehydrated, and embedded undecalcified in methylmethacrylate (J-T Baker, Phillipsburg, NJ, USA). With $50 \mu \mathrm{m}$ intervals, 5 and $8 \mu \mathrm{m}$ thick longitudinal sections were cut using a polycut-E microtome (Reichert-Jung Leica, Heerbrugg, Switzerland), placed on gelatincoated glass slides, deplastified and stained with Goldner’s trichrome. Alkaline phosphatase (ALP) and tartrate-resistant acid phosphatase (TRAP) activity were detected using Naphtol-AS-TR (Sigma-Aldrich) as substrate for both enzymes; Fast Blue BB salt (Sigma-Aldrich) was used as a coupler for ALP. Von Kossa stained sections were used to quantify fat volumes. Fat was identified as area with distinct, translucent ellipsoids in the marrow cavity as previously described. ${ }^{(24)}$ Histomorphometry was performed with a semi-automatic image analyzing system that combined a microscope equipped with a Lucida camera with a digitizing tablet linked to a computer using the OsteoMeasure Software (Osteometrics Inc., Decatur, GA, USA). Osteoblast and osteoclast were identified, and their numbers quantified as previously described. ${ }^{(18)}$ Fluorescence microscopy was used for dynamic histomorphometry. We followed the recommendations of the American Society of Bone and Mineral Research for the nomenclature and abbreviations of the histomorphometric 
parameters. $^{(25)}$

Ex-Vivo Cultures of Bone Marrow Cells. To generate primary cultures of adherent bone marrow cells, we first flushed both tibiae from PIC- and vehicle-treated animals ( $n=6$ per group) by utilizing a 21-gauge needle fixed to a $10 \mathrm{ml}$ syringe filled with Dulbecco’s modified Eagle’s Medium (DMEM) (GIBCO BRL, Gaithersburg, MD, USA). We then filtered the extracted cells from both tibiae by using a 70 $\mu$ nylon mesh cell strainer (BD Bioscience, Bedford, MA, USA). Cells were then combined to reach a volume of $2 \mathrm{ml}$ with approximately $10^{7}$ cells $/ \mathrm{ml}$. Three sets (triplicate) of six-well culture plates were prepared. Four ml of fresh MEM medium and 100- $\mu \mathrm{l}$ aliquot of cell suspension were added to each well. The BMSC were incubated at $37^{\circ} \mathrm{C}$ with $5 \%$ humidified CO2 in MSC growth media. Every 2 to 3 days, the medium was aspirated and replaced with fresh MEM medium to remove all non-adherent cells. Adherent cells were selectively isolated. The MSC were grown up to 80\% confluence for approximately 7 days, defined as MSC at passage 0. Cells were harvested with a mix of $0.25 \%$ trypsin / 1 mM EDTA diluted 1:3 in MSC growth media for $5 \mathrm{~min}$ at $37^{\circ} \mathrm{C}$. Cells were the re-plated and grown to confluence for further expansion. BMSC were used for experiments after passages 2 and 3.

To trigger osteogenic differentiation, $10^{4}$ of the purified cells were diluted in osteogenic medium (prepared with DMEM, 10\% FCS, $0.2 \mathrm{mM}$ dexamethasone, $10 \mathrm{mmol} / \mathrm{L} \beta$ glycerol phosphate and $50 \mu \mathrm{g} / \mathrm{mL}$ ascorbic acid) and plated in six well plates. Culture medium was aspirated and replaced with fresh osteogenic medium twice a week. After 14 days in culture, cells were washed with PBS, ethanol fixed and stained for either alkaline phosphatase (osteoblast differentiation), in which case the cells were counterstained with neutral red (Sigma). A sub-set of differentiating cells were kept for 3 weeks on differentiation media and then stained with alizarin red to assess mineralizing nodules. For alkaline phosphatase quantification, the colonies that had more than $10 \%$ of their cells 
with ALP+AR positive staining were considered as colony forming units osteoblasts (CFU-OB). For quantification of mineralized nodules size, cultures were fixed for 15 min with $10 \%$ formalin, then washed, and stained for 10 to15 min with alizarin red. After the excess dye was washed with distilled water, the remaining, mineralized nodules were labeled as red spots. A Leica microscopy system (Leica DM5500) was used to examine and collect images of the cell cultures. Two independent investigators quantified the size of the nodules by using a flatbed scanner with a builtin a transparency adapter.

For adipogenic differentiation, at $\sim 80 \%$ confluency, BMSC were grown in adipogenic media for up to 21 days to induce the differentiation to adipocytes. The day of induction was recorded as day 0 . Three cycles of alternating adipocyte induction medium (AIM) and adipocyte maintenance medium (AMM) were used for the differentiation. The media were changed every 3 to 4 days. AIM was prepared using Dulbecco Modified Eagle’s Medium (DMEM) which contained 4.5 g/L glucose (SIGMA-Aldrich Pty, Castle Hill, NSW, Australia; cat\# D6429), 10\% Fetal Bovine Serum (FBS), 100 Units/ml penicillin, $100 \mu \mathrm{g} / \mathrm{ml}$ streptomycin sulfate, $0.25 \mu \mathrm{g} / \mathrm{ml}$ amphotericin B (Invitrogen Australia Pty, Mulgrave, VIC, Australia; cat\# 15240-062), 10 g/ml Insulin, 0.2mM Indomethacin, 0.5mM 3-Isobutyl-1-methyl-xanthine and 0.1 $\mu \mathrm{M}$ dexamethasone. AMM is composed of DMEM, $10 \%$ FBS, antibiotic/ antimycotic - consistent with the AIM - and $10 \mu \mathrm{g} / \mathrm{ml}$ of insulin. To assess adipocyte differentiation, ORO staining was used as an indicator of intracellular lipid accumulation. Briefly, the culture medium was removed on day 21 and cells were rinsed once with phosphate buffered saline (PBS). Cells were then fixed with a 10\% formaldehyde in PBS for at least 1 hour. Following this, the fixative was aspirated, and the cells washed with a $60 \%$ isopropanol solution before being left to fully dry. A diluted ORO solution (66.6\%), prepared from a $0.5 \%$ w/v ORO dissolved in isopropanol, was used to stain the cells for 10 minutes at room temperature. The cells 
were then washed 4 times under running tap water. ORO was eluted with $1 \mathrm{ml} 100 \%$ isopropanol for 10 minutes and the absorbance was measured at 500nm

Osteogenic Differentiation of Human MSCs. hMSC are primary cells extracted from bone marrow of young, healthy donors (aged 24-30 years). These cells were purchased from Lonza (Basel, Switzerland). hMSC were seeded in $56 \mathrm{~cm}^{2}$ petri dishes at a density of $5.10^{5} \mathrm{cells} / \mathrm{cm}^{2}$ in mesenchymal stem cell growth media (MSCGM, BulletKit PT-3001, Lonza). hMSC were left growing until they reached $60 \%$ confluence to achieve osteogenesis. Medium was replaced with either MSC growth media or osteoblastogenesis induction medium (OIM) as described above. We changed the medium every 3 days.

Western Blot Analysis. We obtained marrow cells from the left femur of PIC-and vehicle-treated animals ( $n=6-8$ per group) by flushing with DMEM. Red blood cells in marrow cells were hemolyzed using a 0.017 M tris- $\mathrm{HCl}, \mathrm{pH}$ 7.5, 0.8\% ammonium chloride buffer. Hemolyzed bone marrow suspensions were rinsed twice with PBS. Protein extracts were obtained after lysing the cells in 2 volumes of buffer containing 10 mM EDTA, $0.5 \mathrm{mM}$ phenylmethylsulphonyl fluoride, and one tablet of Complete ${ }^{\mathrm{TM}}$ protease inhibitor mixture (Boehringer Mannheim, Laval, Quebec, Canada). Solutions were then centrifuged for $20 \mathrm{~min}$ at $25,000 \times \mathrm{g}$ at $4^{\circ} \mathrm{C}$ and re-suspended in 20 mM HEPES, pH 7.9, 25\% glycerol, 0.42 M NaCl, 1.5 mM MgCl2, 0.2 mM EDTA, 0.5 mM phenylmethylsulphonyl fluoride, and $0.5 \mathrm{mM}$ dithiothreitol. A protein assay kit (Bio-Rad, Mississauga, Ontario) was used to determine the protein levels. Samples were then aliquoted and stored at $-80^{\circ} \mathrm{C}$. For western blot analysis, lysates were dissolved in SDS electrophoresis buffer (Bio-Rad, Hercules, CA, USA), proteins were separated on SDS-polyacrylamide gels and were electrotransfered to polyvinylidene difluoride membranes. The membranes were blocked with a solution of $0.1 \%$ Tween 20 and $10 \%$ non-fat dry milk in PBS and incubated at $4^{\circ} \mathrm{C}$ overnight with 
mouse monoclonal antibodies directed against runt-related transcription factor 2 (Runx2) and osteocalcin (OCN) (1:1000, Santa Cruz Biotechnology, Santacruz, CA, USA).

For quantification of protein expression of $\beta$-catenin-non phospho-ser-45 and GSK-3 $\beta$ in human MSCs, cells were cultured followed by protein extraction and western blotting, which were conducted as outlined above using monoclonal primary antibodies (Santacruz Biotechnology). Sigma secondary antibodies bonded to horseradish peroxidase (1:5000) were used. A chemiluminescence kit from ECL (Amersham, UK) was utilized to detect antigen-antibody complexes. Blots were exposed to high-performance chemiluminescence film (Amersham, UK). The film was scanned, and the optical density of each specific band measured using the Image Master program with intensity expressed as $\mathrm{OD} / \mathrm{mm} 2 / 100 \mu \mathrm{g}$ of total protein. Relative intensity of the samples was determined by comparing the protein of interest in the treated mice with the values of vehicle-treated mice as controls (100\%). The reported values are the average of the samples obtained from 6 mice.

Semi-quantitative Real Time-polymerase Chain Reaction (RT-PCR). The bone marrow cells were flushed and isolated using the method described above. Total RNA was extracted from the cells. Total RNA was extracted from marrow cells using a QIAGEN RNeasy Mini extraction kit according to the manufacturer's instructions (QIAGEN Pty, Doncaster, VIC, Australia). The first strand of cDNA synthesis was performed with $200 \mathrm{ng}$ of RNA, 50 ng of random hexamers and 50 units of reverse transcriptase, and was incubated at $42^{\circ} \mathrm{C}$ for 1 hour, following the manufacturer's instructions (Bioline Australia Pty, Alexandria, NSW, Australia; cat\# BIO-65025).

We used Real-time PCR to detect the expression of the selected genes as markers for osteogenesis. This was performed in duplicate in a total reaction volume of $25 \mu \mathrm{l}$, with $10 \%$ cDNA (or water for non-template control), and $3 \mathrm{mM} \mathrm{MgCl} 2$ and $250 \mathrm{nM}$ of each forward and reverse specific primer 
for target genes and normalizer. Primers for specific detection of mRNA expression included:

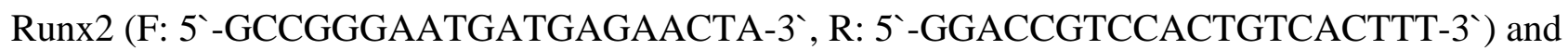
OCN (F: 5’-cTTGGTGCACACCTAGCAGA-3`; 5`ACCTTATTGCCCTCCTGCTT-3`). All PCRs were performed in a Corbett Rotor-Gene 3000 (QIAGEN Pty) using SYBR green with noROX reaction mix and a standard thermal profile as described by supplier (Bioline Australia Pty, Alexandria, NSW, Australia; cat\# QT6750-02). Quantitative RT-PCR data was defined by threshold cycle (Ct) normalized for the housekeeping gene glyceraldehyde-3-phosphate dehydrogenase (GAPDH) (F: 5`AACTTTGGCATTGTGGAAGG-3`, R: 5`ACACATTGGGGGTAGGAACA3).

Quantification of Resorption Pits. Buffy coat blood purchased from the Australian Red Cross, Sydney, Australia, was diluted vol/vol in PBS, then placed over a $15 \mathrm{~mL}$ Ficoll-Paque plus (GE Healthcare Australia, Pty. Ltd.) and centrifuged for 30 minutes at 900g. PBMCs were collected from the interphase and centrifuged for 5 minutes at $400 \mathrm{~g}$. The cell pellet was rinsed and washed in a-MEM medium with $2.2 \mathrm{~g} / \mathrm{L}$ NaHCO3, $0.03 \mathrm{~g} / \mathrm{L}$ penicillin, $0.01 \mathrm{~g} / \mathrm{L}$ streptomycin, and 10\% (v/v) heat-inactivated FBS (10\% $\alpha$-MEM). A layer of mononuclear cells was extracted from the interphase of the PBS and Ficoll-Paque and centrifuged at 400g for 5 minutes. The cell pellet was rinsed and washed in $\alpha$-MEM medium with $2.2 \mathrm{~g} / \mathrm{L} \mathrm{NaHCO3,} 0.03 \mathrm{~g} / \mathrm{L}$ penicillin, $0.01 \mathrm{~g} / \mathrm{L}$ streptomycin, and 10\% (v/v) heat-inactivated FBS (10\% $\alpha$-MEM). A hemocytometer was used to determine the number of mononuclear cells contained in the suspension. PBMCs were seeded at $1 \times$ $10^{6}$ cells/well in $\alpha-$ MEM $10 \%$ FBS in 96-well plates that contained either dentine slices $(4 \times 4$ $\mathrm{mm}$ ) or glass coverslips (5 mm diameter). Following 2 hours of incubation at $37^{\circ} \mathrm{C}$, the cells were washed twice with $\alpha$-MEM that contained 10\% FBS to remove non-adherent cells. Cells were grown in $150 \mu \mathrm{L} \alpha$-MEM with 10\% FBS that contained RANKL (50 ng/ml), human M-CSF (25 
$\mathrm{ng} / \mathrm{ml}$ ) or, only the vehicle. Each treatment was done in triplicate, and the medium was changed every 3 days.

A diamond saw (Struers Accutom 50, Denmark) was used to slice the whale dentine to a thickness of $400 \mu \mathrm{m}$. We treated the dentine slices with acetone and 70\% ethanol for 15-20 minutes. The slices were cleaned for 60 seconds in water using ultra-sound. For sterilization, the slices were passed through a 70\% ethanol bath and rinsed for 10 minutes through milli-Q water, followed by overnight irradiation.

\section{Preparation of Culture on Dentine Slices for Examination by Scanning Electron Microscope.}

Cells were detached from dentine slices by incubation with 0.1 M EDTA for 10 minutes at room temperature, sonicated with $0.25 \mathrm{M}$ ammonium hydroxide for $30 \mathrm{~s}$, passed through graded alcohol to absolute, and air dried. Dentine slices were sputter coated with gold (EMITECH K550X, England) and examined in a JCM-6000 scanning electron microscope (JEOL, MA, USA).

Confocal Microscopy. Four-chamber slides (BD Biosciences) were plated with the human MSCs. When the cells reached an $80 \%$ confluence, osteoblast differentiation was triggered as described above. Cells were fixed, permeabilized and blocked after 8, 12 and 24h following the recommendations for each primary antibody. The primary antibodies were resuspended in a $1 \%$ bovine serum albumin (BSA, Sigma-Aldrich), 0.3\% Triton-X, 0.05\% tween 20 and $1 \mathrm{X}$ trisbuffered saline with anti $\beta$-catenin antibody (Santa Cruz Biotechnology). The hMSCs were incubated with the antibody at $4^{\circ} \mathrm{C}$ overnight under gentle agitation. After washing, the secondary antibody Alexa Fluor ${ }^{\circledR} 488$ Donkey Anti-Rabbit (Invitrogen) was added for $1 \mathrm{~h}$ at room temperature. The cells were then washed and counterstained with diluted (1:5000) nuclear dye 4,6diamidino-2-phenylindole (DAPI) and Phalloidin. Slides were mounted using a buffered glycerol with anti-fade (0.1 M phosphate buffer pH7.4, 90\% glycerol, $5 \mathrm{mg} / \mathrm{ml} \mathrm{n-propyl} \mathrm{gallate).} \mathrm{The} \mathrm{cell}$ 
staining was photographed with a Leica TCS SP5 II confocal microscope using LAS AF Software (Leica Microsystems, Wetzlar, Germany).

Inhibition of Wnts in Human MSCs. hMSCs were grown and differentiated into osteoblasts as

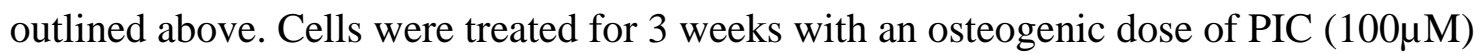
concomitantly with three doses of the inhibitor of WNT Production-3 (IWP-3) (20, 40, 80nM) (Sigma-Aldrich) or vehicle as control. After 3 weeks, hMSCs were fixed and stained with alizarin red as we described earlier. Extraction of the Alizarin red staining using 100mM cetylpyridinium chloride at room temperature for $3 \mathrm{~h}$ was used to quantify the level of matrix mineralization. The absorption level of the extracted Alizarin red S staining was measured at $570 \mathrm{~nm}$. This was normalized to the total amount of protein and expressed as relative units compared with the optical density per mg of protein of control cells.

Statistical Analysis. For each experiment, calculation of the mice sample size was based on a $\beta$ error of 0.2 and an $\alpha$ error of 0.05 using a significant power of more than $90 \%$ to detect a $10 \%$ absolute difference in the parameters of bone formation (Ob.N. and dynamic histomorphometry). The results of the cell culture experiment were expressed as the standard deviation ( \pm SD). Each of the cell culture experiments was performed in triplicate and repeated three times. Levene's test for homogeneity of variances and the unpaired t-test for equality of means for histomorphometry measurements were used to determine differences between the groups. All other variables were compared with a two-way analysis of variance (ANOVA).

\section{Results}

Absorption of Oral PIC in Oophorectomized (OVX) and Sham-Operated Mice. Nine-month- 
old C57Bl/6 mice were either OVX or Sham-operated. Six weeks post-surgery, both groups of animals were treated with three oral doses of PIC $(0.25,0.5,1 \mathrm{~g} / \mathrm{kg} / \mathrm{d})$ in water (Figure $2 \mathrm{~A})$. The concentrations of PIC in serum were measured by gas chromatography/mass spectroscopy (GCMS). Serum concentrations of PIC were increased up to 9-fold in the Sham-operated group and up to 6-fold in the OVX group (Figure 2B).

Therapeutic Safety of Oral PIC in OVX Mice. Table 1 shows the comparison in weight and water consumption between PIC- and vehicle-treated mice. There was no differentiation between the PIC-treated mice and the vehicle-treated animals. The PIC-treated mice survival rate was 100\% with no evidence of any side effects. These combined results suggest that PIC is a safe and welltolerated treatment.

Effect of Oral PIC on Mineral Absorption in OVX Mice. The effect of PIC administration in mice and humans has been associated with increased absorption of minerals in the gut. ${ }^{(21)}$ Since changes in mineral absorption (predominantly calcium magnesium and zinc) could have a direct effect on bone mass, we performed a comprehensive quantification of serum concentrations of nine minerals (Table 2) and duodenal concentrations of $\mathrm{Zn}$ in the gut (Figure 2C) measured by GC-MS. There was no difference in either serum or gut in PIC-treated mice when they were compared to the vehicle-treated controls.

Effect of Oral PIC on Calciotropic Hormones. To determine whether the effects of PIC on bone were not linked with variations in the level of calciotropic hormones, the concentrations of VitD and PTH were determined in the serum of the PIC- and vehicle-treated animals. The serum concentrations of calciotropic hormones in mice treated orally were similar to the vehicle-treated controls (Table 3). 


\section{Treatment of OVX C57Bl6 Mice with PIC Increases Bone Mass and Mechanical Properties}

and Stimulates Bone Formation. Quantitative $\mu \mathrm{CT}$ data (Figure 3A-E and Supplementary Figure 1A-E) indicated a dose-dependent increase in bone relative to the trabecular volume of tissue (BV/TV) in the area of interest of both femur and vertebrae (Supplementary Figure 1B-E), which was reflected in increased number (Tb.N) and thickness (Tb.Th), and decreased separation (Tb.Sp) of the trabeculae in the Sham and OVX mice treated with PIC. This effect was more evident in the Sham-operated group. In addition to the gain in bone mass observed in the trabeculae, $\mu \mathrm{CT}$ data obtained from the femoral midshaft revealed increased cortical BV/TV in the PIC-treated groups (Figure 3E).

In addition, we used the three-point bending technique to evaluate the mechanical performance of the tibia in relation to stiffness, load, and elasticity. Constant force was applied to the midpoint of the tibia diaphysis to assess the maximum load it could bear before fracture or breakage. While PIC had a strong effect on the maximum load the tibias in the treatment groups could withstand, there were differences between groups with the Sham group showing the highest level of response compared to OVX animals (Figure 3F-H). In PIC-treated Sham mice higher force was necessary to break the bones of the control mice than for the OVX animals. In addition, apparent toughness of femoral diaphysis was calculated as apparent toughness divided by mid shaft cortical bone volume fraction. Peak load of the femur diaphysis was strongly and linearly correlated to femur diaphysis cortical BV/TV (Supplemental Figure 1F) $\left(r^{2}=0.24\right.$ for all groups combined), demonstrating treatment effects. Interestingly, these changes in bone structure were associated with a strong reduction in marrow fat demonstrated by a lower ratio of fat to tissue volume (FV/TV) in PIC-treated mice (Figure 4A and B).

Furthermore, higher mineral apposition rates (MAR) in the trabecular and cortical bones of the PIC- 
treated mice compared with the vehicle-treated controls were observed through the use of dynamic histomorphometry performed on tetracycline labeled sections (Figure 5A-E). Increased bone formation corresponded with the higher number of bone forming osteoblasts (N.Ob) in trabecular bone of PIC-treated mice, quantified after normalization to the bone perimeter (Figure 5D). Regarding bone resorption, there were differences in osteoclast numbers (N.Oc) between groups. PIC-treated Sham mice displayed a substantial increase in N.Oc, whereas OVX mice showed no change in N.Oc (Figure 5E). This remained similar to the expected elevation in OVX controls.

\section{Treatment with PIC In Vivo Results in Increased Osteoblastogenesis and Decreased} Adipogenesis in Bone Marrow Stromal Cells (BMSC) Cultured ex vivo. To verify whether the higher number of osteoblasts and lower marrow adipocytes observed in vivo were a consequence of changes in the differentiation capacity of MSCs, BMSC were isolated from bone marrow flushes, then differentiation was induced into either osteoblasts or adipocytes as we previously described. ${ }^{(25)}$ For two weeks, the BMSC were cultured in osteogenic medium before staining with alkaline phosphatase (ALP). At week 3, the cultures were also stained with Alizarin Red for the identification of mineralized nodules. The number of CFU-OB, which is an indicator of the ability of BMSC to differentiate into osteoblasts, was higher in cultures harvested from PIC-treated mice in comparison to those from the vehicle-treated mice (Figure 6A and B). Figure 6C, shows that mineralized nodules in BMSC obtained from PIC-treated mice were larger, thus indicating a higher mineralization potential in BMSC obtained from PIC-treated mice, an effect that was more evident in the OVX group. In addition, BMSC obtained from PIC-treated mice (both Sham and OVX) showed a reduction in their capacity to differentiate into adipocytes (Figure 6A, B and D). 
PIC Induces Osteogenic Transcription Factors in OVX and Sham-Operated Mice. The RTPCR data on the bone marrow cells showed a greater level of expression of the osteogenic genes Runx2 and OCN in all the PIC-treated groups in comparison to the vehicle-treated controls (Figure 7). The analysis of the protein immunoblot from the bone marrow cells showed an increase in the expression of Runx2 (Figure 7B) and OCN (Figure 7E) in PIC-treated groups. Furthermore, to investigate whether the PIC-increased levels of gene and protein expression for Runx2 were also associated with increased Runx2 activity in MSCs, we performed an ELISA analysis of Runx2 complex binding in human MSCs treated with osteogenic concentrations of PIC in vitro. ${ }^{(18)}$ Runx2 shows a higher level of activity in nuclear lysates obtained from MSCs treated with PIC, an effect that lasted up to 24 hours in culture (Figure 7C).

PIC Affects Osteoclast Function in vitro. To gain a better understanding of the observed effect of PIC on bone metabolism in vivo, we tested whether the treatment with PIC acted on osteoclast function. The pit experiments demonstrated that the PIC treatment on osteoclasts derived from PBMCs altered resorption (Figure 7F-G). This indicates that the anabolic and uncoupling effect of PIC on bone involves higher levels of osteoblastogenesis as well as a mild increase in osteoclastogenesis with a reduction in osteoclast function.

Mechanism of Action of PIC in Human MSCs in vitro. After demonstrating that the anabolic effect of PIC in vivo is not associated with changes in $\mathrm{Zn}$ or Ca absorption or indirectly, by affecting serum levels of calciotropic hormones, we then investigated the effect of PIC on main osteogenic pathways that are stimulated by current anabolics. ${ }^{(26)}$ In this case, we focused on the Wnt/ $\beta$-catenin pathway, a common target of most bone anabolics. ${ }^{(26)}$ Human MSCs were induced to differentiate into osteoblasts while treated with osteogenic concentrations of PIC in the media. ${ }^{(18)}$ As shown in Figure 8A, PIC induced higher concentrations of both cytoplasmic and nuclear $\beta$ - 
catenin, an effect that occurred at and earlier timepoint in MSCs treated with PIC. In addition, PIC increased the levels of expression of non-phosphorylated $\beta$-catenin at an earlier time point of differentiation (Figure 8B and C) in osteogenic differentiating MSCs. Furthermore, PIC had no effect on the expression of the glycogen synthase kinase $3 \beta$ (GSK-3 $\beta$ ) (Figure 8B and C), which is an essential negative regulator or "brake” on many anabolic-signaling pathways. This includes Wnt, which suggests that PIC has a Wnt-related role in osteoblastogenesis that is not regulated by GSK$3 \beta$.

To further explore the relationship between PIC and Wnts during osteoblastogenesis, osteogenic differentiating human MSCs were treated with PIC alone or in combination with three different concentrations of IWP-3. As shown in Figure 8D-E, osteogenesis and mineralization were reduced in MSCs treated with IWP-3 alone. Interestingly, this effect was reverted by PIC, thus suggesting that PIC has an anabolic effect on bone when Wnts are absent, while still activating the anabolic elements of the Wnt canonical pathways.

\section{Discussion}

This study has characterized the bone phenotype of skeletally mature OVX and Sham-operated mice after pharmacological treatment with PIC. Our results demonstrate that PIC increases bone formation while also partially affecting bone resorption. We showed that this osteoanabolic effect was partly due to increased differentiation of bone marrow precursor cells into mature osteoblasts. The bone mass was increased concomitant with a marked reduction in adipogenesis and marrow fat infiltration. This was associated with a higher transcription of osteogenic genes and activation of the Wnt/ $\beta$-catenin pathway; mimicking the effect of Wnts on bone. 
PIC is a pyridine molecule with a carboxyl side chain in position 2. It is an isomer of nicotinic acid and has chelating capacity on several minerals, which include chromium and zinc. PIC is highly water soluble and is well tolerated, even at high doses. ${ }^{(22)}$ Chromium PIC has previously been proposed as a potential treatment for metabolic syndrome and several neurodegenerative diseases. ${ }^{(27-29)}$ During normal aging, there is a decrease in tryptophan levels and IDO-1 expression in all tissues, which could be associated with lower bioavailability of PIC. ${ }^{(30)}$ Generally, reported physiological PIC concentrations are within the low to mid nanomolar range. The doses used in our study, which have been used previously in other murine models, ${ }^{(21,22)}$ are between $6-9$ fold the physiological PIC concentrations observed in control mice and closely correspond to previous osteogenic doses in vitro. Importantly, these increased serum concentrations of PIC in both groups were not associated with side effects in our treated animals. In addition, although oophorectomy did not affect physiological concentrations of PIC, therapeutic concentrations of PIC were much lower in the OVX mice confirming previous reports on the regulatory role of estrogens in KYN pathway $\operatorname{activity.}^{(31)}$

PIC is one of the end-products of the KYN pathway following activation of IDO-1, which is induced primarily by the proinflammatory cytokine IFN $\gamma$. Interestingly, the observed anabolic effect of PIC on OVX mice shows similar features than our previously reported anabolic effect of IFN $\gamma$ on the same mouse model where IFN $\gamma$ stimulated osteoblastogenesis and inhibited adipogenesis. ${ }^{(32,33)}$ Although the mechanism(s) explaining the anabolic effect of IFN $\gamma$ on bone remain unclear, it is tempting to hypothesize that in the absence of inflammation, which has deleterious effect on bone mass, activation of IDO-1 by IFN $\gamma$ induces high levels of PIC, which is followed by an increase in osteoblastogenesis and bone formation. 
However, not all end products of the KYN pathway have a beneficial effect on bone formation, KYN is a compound produced in the first step of tryptophan catabolism through the KYN pathway. KYN exhibits the ability to stop BMSCs proliferation and osteogenic differentiation. In addition, increasing levels of pathogenic KYN due to aging and the inhibition of anabolic signals leads to the development of osteoporosis. ${ }^{(34,35)}$

In contrast, our experiments found that PIC has a strong anabolic effect that is evident not only in OVX but also in Sham-operated mice. This effect included a gain in bone mass and bone formation in both trabecular and cortical bone; a response that occurs with just a few osteoanabolics and that is mediated through effects on the Wnt/ $\beta$-catenin pathway. ${ }^{(36)}$ Overall, PIC demonstrated the majority of the effects expected from a strong osteoanabolic, along with the lack of side effects in the short term. Although this was more evident in Sham-operated mice, PIC showed strong effects on bone formation in both models, which were also associated with a decrease in marrow fat that could also have a beneficial effect by reducing local lipotoxicity. ${ }^{(37)}$ Additionally, PIC demonstrated an improved effect on cortical bone, which, if translated in humans, could have an improved effect on reducing the risk of hip fractures. PIC also demonstrated an effect on bone strength and a mechanism of action that sensibly concurs with those observed in other osteoanabolics. ${ }^{\left({ }^{8}\right)}$ A major advantage of PIC compared to other osteoanabolics is that it could be administered orally, which would facilitate administration in humans, especially those at high risk of osteoporotic fractures such as frail older individuals where administration of a daily or monthly injection of a bone anabolic has been challenging.

However, several questions remain unanswered including the potential side effects after long-term use of PIC in this, and other animal models (i.e. normally aged mice); the effect of PIC on fracture 
healing; and the potential effect on muscle; which in humans, would have a combined effect against falls and fractures. All these questions will be resolved in future studies in order to complete the preclinical validation of PIC as a new osteoanabolic.

The observed differences in response to PIC between OVX and Sham-operated groups were mostly expected due to the effect of OVX on bone. Nevertheless, although OVX mice showed higher levels of marrow fat and very reduced bone quality, treatment with PIC was able to revert those features, although at a lower degree, compared to its effect on Sham-operated mice mostly with a dose-dependent effect. However, the therapeutic response to PIC seems to reach a plateau at a very high dose $(1 \mathrm{~g} / \mathrm{Kg} / \mathrm{d})$ with improvement in several parameters of bone formation and bone quality being similar to those observed with a lower dose $(0.5 \mathrm{~g} / \mathrm{Kg} / \mathrm{d})$. This correlates with serum levels of PIC obtained in mice receiving $1 \mathrm{~g} / \mathrm{Kg} / \mathrm{d}$, which were similar to those in mice receiving $0.5 \mathrm{~g} / \mathrm{Kg} / \mathrm{d}$. This has been observed in other osteoporosis treatments. ${ }^{(38)}$ Nevertheless, further experiments evaluating the pharmacokinetics and pharmacodynamics of PIC are still required.

Regarding the mechanism of action of PIC in osteoporotic mice, we performed a comprehensive assessment of the effect of PIC in bone and other organs both in vivo and in vitro. The most widely known physical characteristic of PIC is its efficient chelator properties for a range of metals including $\mathrm{Ni}, \mathrm{Zn}, \mathrm{Cd}, \mathrm{Pb}$ and $\mathrm{Cu} .{ }^{(39)}$ Considering that $\mathrm{Zn}$ and $\mathrm{Ca}$ are required in bone formation, we investigated whether PIC affected serum levels of these and other essential metals as well as local levels of $\mathrm{Zn}$ in the gut. Our experiments demonstrated that treatment with PIC did not affect serum and tissue levels of these metals. Furthermore, the lack of effect of PIC on calcium levels was also demonstrated by the steady levels of serum vitamin D and PTH in all treated groups.

We then evaluated whether, as other osteoanabolics, ${ }^{(38)} \mathrm{PIC}$ had an effect on the canonical Wnt/ $\beta$ - 
catenin pathway. Using an in vitro model of human MSC differentiation, we found that concurring with other osteoanabolics, PIC induces an early peak and rapid increase of nuclear and cytoplasmic levels of active $\beta$-catenin, an effect that was also associated with an increase in the expression of osteogenic factors Runx2 and OCN. We then investigated whether PIC has a direct effect on Wnts by using a strong Wnt inhibitor (IWP-3). ${ }^{(40)}$ As expected, treatment with IWP-3 inhibited osteoblastogenesis. Interestingly, PIC reverted this effect thus suggesting that PIC has a Wnt-like effect on bone that is independent of the presence of Wnts in the differentiating cells, thus making this mechanism of action unique to this compound compared to other osteoanabolics that predominantly exert their action via direct activation of Wnts. ${ }^{(36)}$

In summary, we believe that we have identified PIC as a compound with a high potential to become a novel anabolic treatment for osteoporosis in the future. PIC demonstrated to be safe and highly effective as an osteoanabolic in our mouse models. With current osteoporosis treatments limited by unpleasant side effects associated with their long-term use, and with most only stopping bone resorption rather than building new bone, new treatments for this debilitating condition are urgently required. ${ }^{(38)}$ Nevertheless, further research studies are needed to assess pharmacokinetics, pharmacodynamics, and dosing (i.e. lower or intermittent doses) before PIC could be considered a drug candidate suitable for development as a new human therapeutic for osteoporosis. 


\section{Acknowledgements}

This study was supported by a Commercial Development \& Industry Partnerships (CDIP) grant from Sydnovate (The University of Sydney), and seed grants from the Nepean Medical Research Foundation and the Australian Institute for Musculoskeletal Science (AIMSS) to CV, AAS and GD. CV was funded by a grant from the Rebecca L. Cooper Foundation. GJG is funded by grants from the Australian National Health and Medical Research Council (NHMRC), Australian Research Council (ARC) and Macquarie University. The authors would like to thank Prof. Nicholas Hunt from the Kolling Institute (Sydney, Australia) for his scientific advice and Dr. Sara Vogrin for her assistance with the statistical analyses.

\section{Author's Roles}

Study design: GD and CV. Study conduct: GD, CV, WL, AAS, MK, CKL, DEM and GG. Data collection: CV, WL, AAS, CKL, and DEM. Data analysis: GD, CV, WL, AAS, and CKL. Data interpretation: GD, CV, WL, AAS, MK, CKL, DEM and GG. Drafting manuscript: GD, CV, WL, and GG. Revising manuscript content: GD, CV, WL, AAS, MK, CKL, DEM and GG. Approving final version of manuscript: GD, CV, WL, AAS, MK, CKL, DEM and GG. GD takes responsibility for the integrity of the data analysis.

This article is protected by copyright. All rights reserved. 


\section{Conflict of Interest}

No conflict of interest to declare.

\section{References}

1. Li G, Thabane L, Papaioannou A, Ioannidis G, Levine MA, Adachi JD. An overview of osteoporosis and frailty in the elderly. BMC Musculoskelet Disord. 2017;18(1):46.

2. Bliuc D, Center JR. Determinants of mortality risk following osteoporotic fractures. Curr Opin Rheumatol. 2016;28(4):413-9

3. Saito T, Sterbenz JM, Malay S, Zhong L, MacEachern MP, Chung KC. Effectiveness of antiosteoporotic drugs to prevent secondary fragility fractures: systematic review and meta-analysis. Osteoporos Int. 2017;28(12):3289-3300

4. Harsløf T, Langdahl BL. New horizons in osteoporosis therapies. Curr Opin Pharmacol. 2016;28:38-42

5. No authors listed. Amgen and UCB announce increased cardiovascular risk in patients receiving romosozumab, an anti-sclerotin antibody. Rheumatology (Oxford). 2017;56:e21.

6. Drake MT, Clarke BL, Oursler MJ, et al. Cathepsin K Inhibitors for Osteoporosis: Biology, Potential Clinical Utility, and Lessons Learned. Endocr Rev. 2017;38:325-350.

7. Michalowska M, Znorko B, Kaminski T, Oksztulska-Kolanek E, Pawlak D. New insights into 
tryptophan and its metabolites in the regulation of bone metabolism. J Physiol Pharmacol. 2015;66(6):779-91.

8. Ducy P, Karsenty G. The two faces of serotonin in bone biology. J Cell Biol. 2010;191:7-13.

9. Ducy P. 5-HT and bone biology. Curr Opin Pharmacol. 2011;11:34-8.

10. Karsenty G, Yadav VK. Regulation of bone mass by serotonin: molecular biology and therapeutic implications. Annu Rev Med. 2011;62:323-31.

11. Michalowska M, Znorko B, Kaminski T, et al. New insights into tryptophan and its metabolites in the regulation of bone metabolism. J Physiol Pharmacol. 2015;66:779-91.

12. Yadav VK, Balaji S, Suresh PS, et al. Pharmacological inhibition of gut-derived serotonin synthesis is a potential bone anabolic treatment for osteoporosis. Nat Med. 2010;16:308-12.

13. Goltzman D. LRP5, serotonin, and bone: complexity, contradictions, and conundrums. J Bone Miner Res. 2011;26:1997-2001.

14. Guillemin GJ. Quinolinic acid, the inescapable neurotoxin. FEBS J. 2012;279:1356-65

15. Braidy N, Grant R. Kynurenine pathway metabolism and neuroinflammatory disease. Neural Regen Res. 2017;12:39-42.

16. Vécsei L, Szalárdy L, Fülöp F, et al. Kynurenines in the CNS: recent advances and new questions. Nat Rev Drug Discov. 2013;12:64-82.

17. Jones SP, Guillemin GJ, Brew BJ. The Kynurenine Pathway in Stem Cell Biology. Int J Tryptophan Res. 2013;6:57-66.

18. Vidal C, Li W, Santner-Nanan B, Lim CK, Guillemin GJ, Ball HJ, Hunt NH, Nanan R, Duque G. The kynurenine pathway of tryptophan degradation is activated during osteoblastogenesis. Stem Cells. 2015;33:111-21.

19. Apalset EM, Gjesdal CG, Ueland PM, et al. IFN $\gamma$ mediated inflammation and the kynurenine 
pathway in relation to bone mineral density. Clin Exp Immunol. Clin Exp Immunol. 2014;176:452-60.

20. Forrest CM, Mackay GM, Oxford L, et al. Kynurenine pathway metabolism in patients with osteoporosis after 2 years of drug treatment. Clin Exp Pharmacol Physiol. 2006;33:1078-87.

21. Seal CJ, Heaton FW. Effect of dietary picolinic acid on the metabolism of exogenous and endogenous zinc in the rat. J Nutr. 1985;115:986-93.

22. Lapin IP. Antagonism of Kynurenine-induced seizures. J Neural Transmission 1983;56:177-85.

23. Bouxsein ML, Boyd SK, Christiansen BA, Guldberg RE, Jepsen KJ, Müller R. Guidelines for assessment of bone microstructure in rodents using micro-computed tomography, J Bone Miner Res. 2010;25:1468-86.

24. Duque G, Li W, Vidal C, Bermeo S, Rivas D, Henderson J. Pharmacological inhibition of PPAR $\gamma$ increases osteoblastogenesis and bone mass in male C57BL/6 mice. J Bone Miner Res. 2013;28(3):639-48

25. Dempster DW, Compston JE, Drezner MK, et al. Standardized nomenclature, symbols, and units for bone histomorphometry: a 2012 update of the report of the ASBMR Histomorphometry Nomenclature Committee. J Bone Miner Res. 2013;28(1):2-17.

26. Russow G, Jahn D, Appelt J, Märdian S, Tsitsilonis S, Keller J. Anabolic Therapies in Osteoporosis and Bone Regeneration. Int J Mol Sci. 2018;20(1).

27. Guillemin GJ, Cullen KM, Lim CK, et al. Characterization of the kynurenine pathway in human neurons. J Neurosci. 2007;27:12884-92.

28. Grant RS, Coggan SE, Smythe GA. The physiological action of picolinic Acid in the human brain. Int J Tryptophan Res. 2009;2:71-9.

29. Power M, Pratley R. Alternative and complementary treatments for metabolic syndrome. Curr 
Diab Rep. 2011;11:173-78.

30. Braidy N, Guillemin GJ, Mansour H, Chan-Ling T, Grant R. Changes in kynurenine pathway metabolism in the brain, liver and kidney of aged female Wistar rats. FEBS J 2011; 278:4425-34.

31. de Bie J, Lim CK, Guillemin GJ. Kynurenines, Gender and Neuroinflammation; Showcase Schizophrenia. Neurotox Res. 2016;30:285-94.

32. Duque G, Huang DC, Dion N, et al. Interferon- $\gamma$ plays a role in bone formation in vivo and rescues osteoporosis in ovariectomized mice. J Bone Miner Res. 2011;26(7):1472-83.

33. Vidal C, Bermeo S, Li W, Huang D, Kremer R, Duque G. Interferon gamma inhibits adipogenesis in vitro and prevents marrow fat infiltration in oophorectomized mice. Stem Cells. 2012;30(5):1042-8.

34. Refaey ME, McGee-Lawrence ME, Fulzele S, et al. Kynurenine, a Tryptophan Metabolite That Accumulates With Age, Induces Bone Loss. J Bone Miner Res. 2017;32(11):2182-93

35. Kim BJ, Hamrick MW, Yoo HJ, et al. The Detrimental Effects of Kynurenine, a Tryptophan Metabolite, on Human Bone Metabolism. J Clin Endocrinol Metab. 2019;104(6):2334-42.

36. Li J, Bao Q, Chen S, et al. Different bone remodeling levels of trabecular and cortical bone in response to changes in Wnt/ $\beta$-catenin signaling in mice. J Orthop Res. 2017;35(4):812-19.

37. Singh L, Tyagi S, Myers D, Duque G. Good, Bad, or Ugly: the Biological Roles of Bone Marrow Fat. Curr Osteoporos Rep. 2018;16(2):130-7.

38. Khosla S, Hofbauer LC. Osteoporosis treatment: recent developments and ongoing challenges. Lancet Diabetes Endocrinol. 2017;5(11):898-907.

39. Suzuki K, Yasuda M, Yamasaki K. Stability constants of picolinic and quinaldic acid chelates of bivalent metals. J Phys Chem. 1957;61:229-31.

40. Chen B, Dodge ME, Tang W, et al. Small molecule-mediated disruption of Wnt-dependent 
signaling in tissue regeneration and cancer. Nat Chem Biol. 2009;5:100-7.

\section{Tables}

Table 1. Weight comparison between groups after 6 weeks of treatment. Water left in the bottle (initial volume of $500 \mathrm{~mL}$ ) was also quantified every week. The table shows the average of water

This article is protected by copyright. All rights reserved. 
volumes left over every week for the whole duration of the treatment. These volumes correspond with the drinking pattern previously reported in this mouse model.

\begin{tabular}{|l|c|c|c|}
\hline Group (treatment-dose in g/kg/d) & Weight $(\mathbf{g m}) \pm$ SD & Water left (ml/week) \pm SD & $\boldsymbol{p}$ \\
\hline Sham control & $26 \pm 1.3$ & $175 \pm 25$ & 0.19 \\
\hline Sham (PIC 0.25) & $27 \pm 2.0$ & $200 \pm 32$ & 0.15 \\
\hline Sham (PIC 0.5) & $26 \pm 2.4$ & $155 \pm 35$ & 0.35 \\
\hline Sham (PIC 1) & $26 \pm 1.3$ & $260 \pm 45$ & 0.13 \\
\hline OVX Control & $28 \pm 1.9$ & $175 \pm 32$ & 0.18 \\
\hline OVX (PIC 0.25) & $26 \pm 1.6$ & $195 \pm 28$ & 0.20 \\
\hline OVX (PIC 0.5) & $28 \pm 0.4$ & $195 \pm 35$ & 0.26 \\
\hline OVX (PIC 1) & $26 \pm 1.3$ & $250 \pm 38$ & 0.13 \\
\hline
\end{tabular}

Table 2. Serum concentration $( \pm S D$ ) of nine essential minerals in PIC- vs. vehicle-treated mice. No difference was found in any of the measured minerals between the eight groups.

\begin{tabular}{|l|c|c|c|c|c|c|c|c|}
\hline \multicolumn{1}{|c|}{ Mineral } & $\begin{array}{c}\text { Sham } \\
\text { control }\end{array}$ & $\begin{array}{c}\text { Sham } \\
\text { (PIC } \\
\mathbf{0 . 2 5}\end{array}$ & $\begin{array}{c}\text { Sham } \\
\text { (PIC 0.5) }\end{array}$ & $\begin{array}{c}\text { SHAM } \\
\text { (PIC } \\
\mathbf{1}\end{array}$ & $\begin{array}{c}\text { OVX } \\
\text { control }\end{array}$ & $\begin{array}{c}\text { OVX } \\
\text { (PIC } \\
\mathbf{0 . 2 5}\end{array}$ & $\begin{array}{c}\text { OVX } \\
\text { (PIC 0.5) }\end{array}$ & $\begin{array}{c}\text { OVX } \\
\text { (PIC 1) }\end{array}$ \\
\hline Carbon $(\mathrm{mEq} / \mathrm{L})$ & $30 \pm 0.2$ & $29 \pm 0.9$ & $28 \pm 0.2$ & $31 \pm 1.2$ & $30 \pm 0.8$ & $30 \pm 0.5$ & $29 \pm 1.2$ & $28 \pm 1$ \\
\hline Magnesium $(\mathrm{mg} / \mathrm{dL})$ & $3 \pm 0.3$ & $2.8 \pm 0.4$ & $2.8 \pm 0.25$ & $2.8 \pm 0.22$ & $2.9 \pm 0.5$ & $2.6 \pm 0.35$ & $2.4 \pm 0.17$ & $2.6 \pm 0.2$ \\
\hline Aluminum $(\mu \mathrm{mol} / \mathrm{L})$ & $4 \pm 2$ & $7 \pm 3$ & $4 \pm 1$ & $5 \pm 2$ & $4 \pm 2$ & $2 \pm 1.4$ & $2.5 \pm 0.2$ & $4.5 \pm 3$ \\
\hline Calcium $(\mathrm{mg} / \mathrm{dl})$ & $9.2 \pm 0.4$ & $9.1 \pm 0.05$ & $8.9 \pm 0.28$ & $9.1 \pm 0.24$ & $9.4 \pm 0.42$ & $9 \pm 0.25$ & $8.9 \pm 0.27$ & $8.7 \pm 0.07$ \\
\hline $\begin{array}{l}\text { Manganese } \\
(\mu \mathrm{g} / \mathrm{L})\end{array}$ & $4 \pm 2$ & $4 \pm 1.6$ & $3 \pm 0.5$ & $4 \pm 0.9$ & $3.6 \pm 1$ & $3 \pm 0.4$ & $5 \pm 2.5$ & $3 \pm 0.6$ \\
\hline Iron $(\mu \mathrm{g} / \mathrm{dL})$ & $200 \pm 20$ & $240 \pm 70$ & $200 \pm 80$ & $200 \pm 40$ & $180 \pm 50$ & $170 \pm 30$ & $200 \pm 70$ & $180 \pm 20$ \\
\hline Cobalt $(\mu \mathrm{g} / \mathrm{L})$ & $2.3 \pm 0.3$ & $2.4 \pm 0.4$ & $2.1 \pm 0.2$ & $2.2 \pm 0.1$ & $2.2 \pm 0.4$ & $2 \pm 0.1$ & $2 \pm 0.2$ & $1.9 \pm 0.1$ \\
\hline Copper $(\mu \mathrm{g} / \mathrm{L})$ & $619 \pm 19$ & $643 \pm 52$ & $576 \pm 69$ & $703 \pm 56$ & $622 \pm 7$ & $647 \pm 36$ & $631 \pm 10$ & $614 \pm 31$ \\
\hline Zinc $(\mu \mathrm{g} / \mathrm{dL})$ & $73 \pm 2.1$ & $63 \pm 5.2$ & $62 \pm 7.4$ & $69 \pm 9.3$ & $67 \pm 1.6$ & $72 \pm 6$ & $69 \pm 7.7$ & $73 \pm 2.4$ \\
\hline
\end{tabular}


Table 3. Serum concentrations ( \pm SD) of calciotropic hormones (vitamin D and PTH) in PIC- vs.

vehicle-treated mice. All groups showed similar serum concentrations of the calciotropic hormones.

\begin{tabular}{|l|c|c|}
\hline Group (treatment-dose in g/Kg/d) & $\begin{array}{c}\text { Vitamin D } \\
\text { (nmol/L) }\end{array}$ & $\begin{array}{c}\text { PTH } \\
\text { (pg/ml) }\end{array}$ \\
\hline Sham control & $52 \pm 13$ & $36 \pm 6$ \\
\hline Sham (PIC 0.25) & $54 \pm 12$ & $40 \pm 3$ \\
\hline Sham (PIC 0.5) & $52 \pm 14$ & $35 \pm 5$ \\
\hline Sham (PIC 1) & $53 \pm 13$ & $36 \pm 5$ \\
\hline OVX Control & $56 \pm 19$ & $34 \pm 6$ \\
\hline OVX (PIC 0.25) & $52 \pm 16$ & $35 \pm 8$ \\
\hline OVX (PIC 0.5) & $56 \pm 4$ & $35 \pm 6$ \\
\hline OVX (PIC 1) & $52 \pm 13$ & $40 \pm 2$ \\
\hline
\end{tabular}

This article is protected by copyright. All rights reserved. 


\section{Figure legends}

Fig. 1. The kynurenine pathway of tryptophan degradation.

Fig. 2. Pharmacokinetics of Oral PIC in OVX and Sham-Operated Mice. (A) Study design; (B) Mice treated with oral PIC showed higher serum concentrations of PIC as compared with vehicletreated animals. All PIC-treated OVX mice showed lower serum concentrations of PIC than their Sham-operated counterparts. The higher dose of PIC $(1 \mathrm{~g} / \mathrm{Kg} / \mathrm{d})$ was not associated with higher serum concentrations. Values are presented as median and interquartile range. $n=10$ per group. (C) Tissue concentrations of $\mathrm{Zn}$ in the duodenum of Sham and OVX mice treated with either PA or vehicle. No difference was found between the eight groups, indicating that the therapeutic effect of PA on bone occurs separately to $\mathrm{Zn}$ absorption in the gut. Values are presented as median and interquartile range. $n=10$ per group.

Fig. 3. PIC Increases Bone Mass in Both Sham-Operated and OVX Mice. (A) Quantitative $\mu C T$ data indicated a PIC-induced increase in trabecular bone relative to tissue volume (BV/TV) in the region of interest or femur (F) (B), which was shown in both higher numbers (Tb.N) (C) and thickness (Tb.Th) (D) in PIC-treated Sham and OVX mice. This effect was also observed in the midshaft area (mostly cortical) Ct. BV/TV (E) of mice treated with PIC. Values are presented as median and interquartile range. $n=6-8$ per group. (F-H) Effect of PIC therapy on the mechanical strength of tibia. Stiffness (resistance of bone to displacement) (F) and Maximum Load (G) and Extension were quantified $\mathbf{( H )}$. In all measurements, the lower doses of PIC improved strength in 
Sham-operated mice and reverted the effect of OVX on bone. Values are presented as median and interquartile range. Data are shown as mean \pm SD. $n=10$ per group.

Fig. 4. PIC Reduces Bone Marrow Adipose Tissue in Sham-Operated and OVX Mice. (A) Von Kossa stained sections were used to quantify fat volumes. Fat was identified as area with distinct, translucent ellipsoids in the marrow cavity. The higher levels of fat in filtration within the bone marrow were observed in OVX mice. (B) Treatment with PIC markedly reduced marrow fat (fat volume/total volume, BV/TV) in both Sham-operated and OVX mice. Values are presented as median and interquartile range. Micrographs represent 6-8 different mice from each treatment group. Magnification x20.

Fig. 5. PIC Induces Bone Formation in Sham-Operated and OVX Mice. (A) Fluorochrome labeling of bone turnover in distal femur indicated an increase in bone turnover (double labeling, arrows) in trabecular (left panels) and cortical (right panels) bone of the PIC-treated Sham and OVX mice as demonstrated by an increase in apposition rates (Aj.AR) in PIC-treated animals (B and C). Micrographs represent 6-8 different mice from each treatment group. Magnification x20. (D) Staining showed that PIC-treated mice have higher osteoblast number per bone perimeter (N.Ob/B.Pm). (E) Osteoclast number (N.Oc/E.Pm) was higher in OVX mice and was increased by PIC treatment in the Sham-operated group. Values are presented as median and interquartile range. $n=6-8$ per group.

\section{Fig. 6. PIC Increases Osteoblastogenesis Ex Vivo in Sham-Operated and OVX Mice.}

(A-B) After 2 weeks of culture in osteogenic media, ex vivo cultures of bone marrow stromal cells (BMSC) obtained from mice tibiae demonstrated that both Sham (A) and OVX (B) mice treated with PIC generated higher number of bone forming units (ALP: Alkaline Phosphatase, AR: Alizarin red, ALP+NR: ALP + neutral red) as compared to the untreated controls. (C) After 3 weeks of 
culture in osteogenic media BMSC obtained from PIC-treated mice have a higher capacity to mineralize as demonstrated by a larger average nodule size in BMSC obtained from PIC-treated mice. Each data point represents the average of one independent experiment. Values were presented as median and interquartile range. (D) After 3 weeks of culture in adipogenic media, ex vivo cultures of BMSC obtained from mice tibiae (Panels A and B lower panels; ORO: Oil red O) showed that PIC-treated Sham and OVX mice generated lower lipid accumulation compared to the untreated controls. Each data point represents the average of one independent experiment. Values were presented as median and interquartile range. $n=6$ per group.

Fig. 7. Effects of PIC Treatment on Osteogenic Genes. (A-E) mRNA and total protein were obtained from bone marrow flushed from tibiae of PIC- and vehicle-treated animals. Treatment with PIC affects transcription of both Runx2 and osteocalcin (OCN) as indicated by lower levels of gene expression after correction with housekeeping gene (GAPDH) (A) and (D) respectively. Further, PA also affects Runx2 (B) and OCN translation (E) measured by western blot. The relative intensity to these proteins is presented in the bar graph as a ratio of $\beta$-tubulin expression. Each data point represents the average of one independent experiment ( $n=6-8$ per group). Values are presented as median and interquartile range. Statistical significance was calculated compared with their control group. (C) Effect of PA on Runx2 activity in human mesenchymal stem cells (MSCs). Runx2 activity in nuclear lysates obtained from hMSCs treated with PIC or vehicle, was determined using an ELISA based ALM3-activity kit. Each data point represents the average of one independent experiment ( $n=6-8$ per group). Values are presented as median and interquartile range. Statistical significance was calculated compared with their control group. (F-G) Human OCs treated with increasing doses of PIC in vitro achieves increased erosion by generating more \%pit surface/bone surface (BS). Data is calculated from three individual experiments. Values are presented as median 
and interquartile range.

\section{Fig. 8. The osteoanabolic effect of PIC is exerted via activation of the $\beta$-catenin/Wnts}

pathway. (A) Human mesenchymal stem cells (hMSCs) were treated with PIC or vehicle control (OIM + vehicle) for 8, 12 and 24 hours. Cells were fixed and stained using rabbit anti- $\beta$-catenin antibody, followed by goat anti-rabbit Alexa 555 secondary antibody and counterstained with Dapi and phalloidin. Images were captured using epi-fluorescence microscopy. PIC-treated cells showed higher accumulation of nuclear and cytoplasmic $\beta$-catenin. Micrographs represent cells from five different plates from each treatment group. Magnification x20. (B) hMSCs were treated with PA or vehicle control for 8, 12 and 24, 48 and 72 hours. Proteins were extracted using RIPA buffer and resolved under reducing conditions on $10 \%$ Tris-Glycine gels. This was followed by immunoblotting on PVDF membrane (0.2 micron). Membranes were probed for stabilized beta catenin (non-phosphor ser45), and for GSK3-beta, a downstream inhibitor of $\beta$-catenin stability. Representative images from three individual experiments $(n=3)$. (C) Densitometric analysis of western blots obtained from chemiluminescent imaging of immunoblots probed for the presence of $\beta$-catenin-non-phosphor ser45 and GSK3 $\beta$ captured using Fusion-FX ${ }^{\circledR}$ imager with a CCD cooled camera. Analysis was performed using ImageJ®. Signal was normalized with GAPDH as a housekeeping protein. Representative images from three individual experiments $(n=3)$. Values are presented as median and interquartile range. (D) Reversal of IWP3-induced decrease in osteogenesis in hMSCs by treatment with anabolic concentration of PIC in vitro. hMSCs were cultured up to day 14 under various conditions and stained with alizarin red. Representative results from three individual experiments $(n=3)$. (E) PIC reverts the inhibitory effect of IWP3 on osteoblastogenesis. The figure shows spectrophotometric quantification of alizarin red staining in the respective groups. Experiments were repeated three times. Values are presented as median and 
interquartile range.

Supplementary Figure 1. (A) Quantitative $\mu \mathrm{CT}$ data indicated a PIC-induced increase in separation (Tb.Sp) in femur. PIC also induced an increase in trabecular bone relative to tissue volume (BV/TV) (B) in the region of interest or vertebrae (F), which was shown in higher numbers (Tb.N) (C), lower separation (Tb.Sp) (D), and higher thickness (Tb.Th) (E) in PIC-treated Sham and OVX mice (illustrative images in Fig. 3). Values are presented as median and interquartile range. $n=6-8$ per group. (E) Scatter plot of peak load versus cortical BV/TV (Ct BV/TV) in femoral diaphysis. Individual group $\mathrm{r}^{2}$ values ranged from 0.18-0.42; all $p<0.05$. 


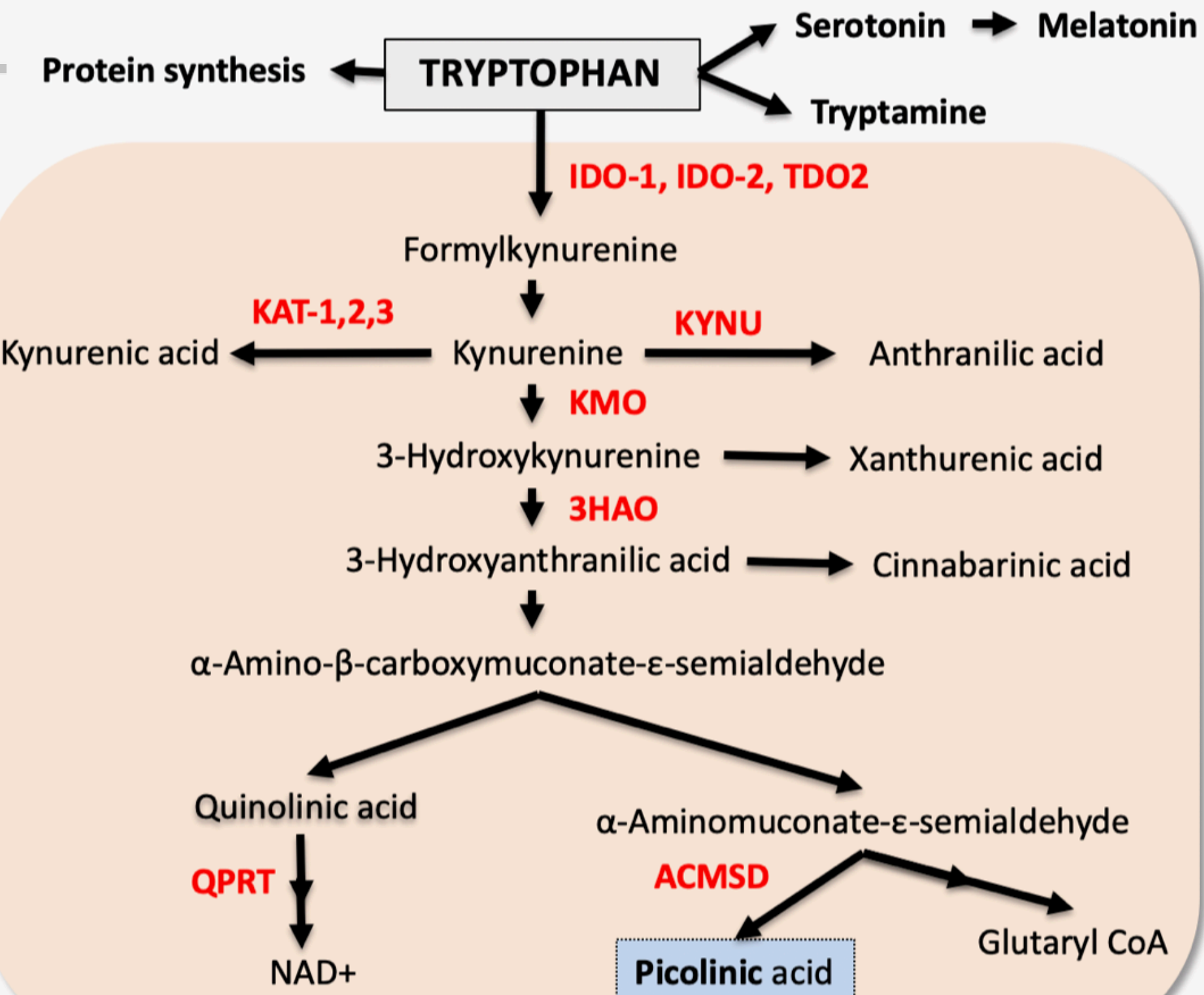

JBMR_4125_Figure 1.tiff 

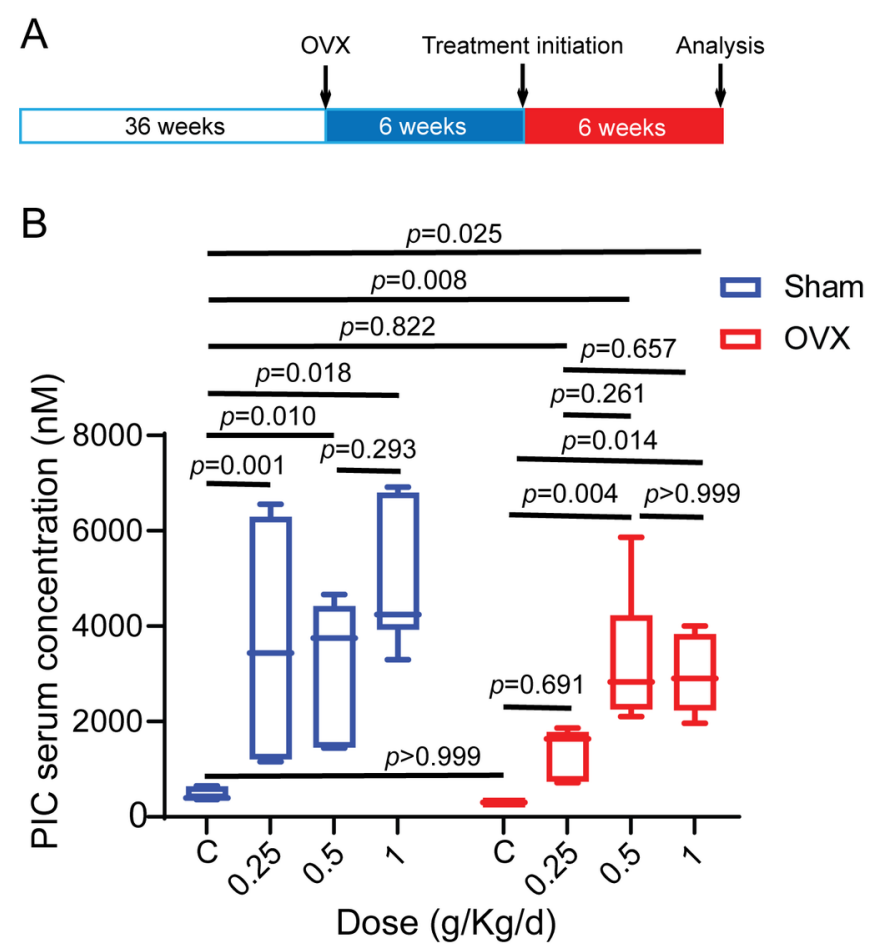

C

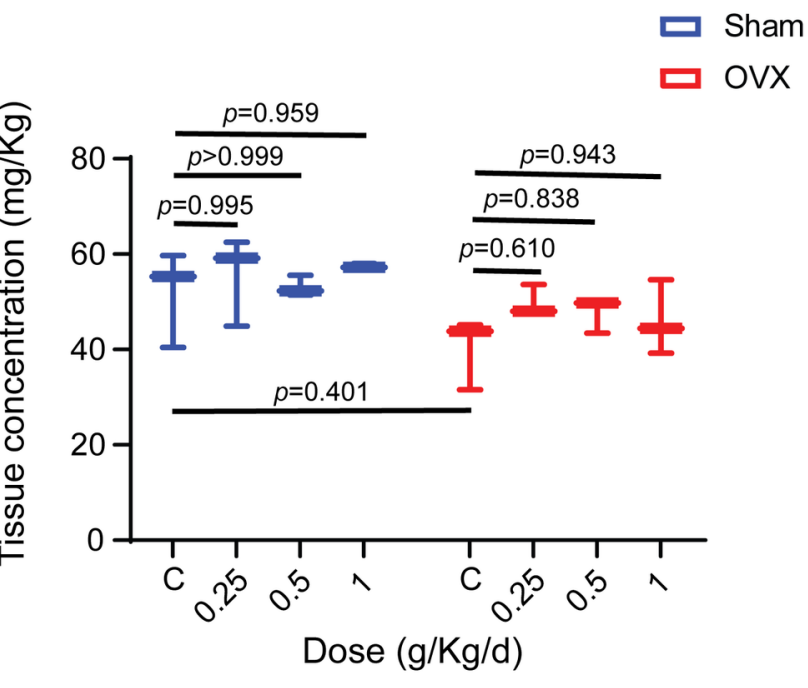

JBMR_4125_Figure 2.tif 

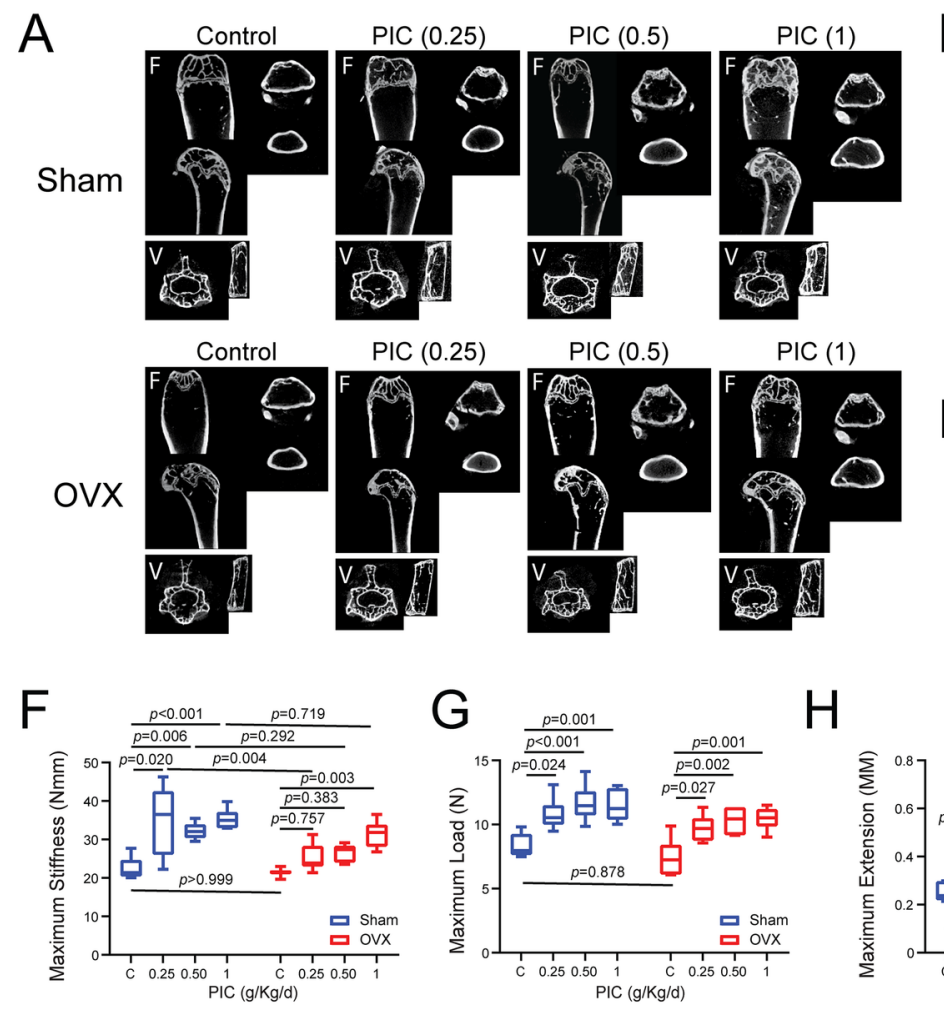

H
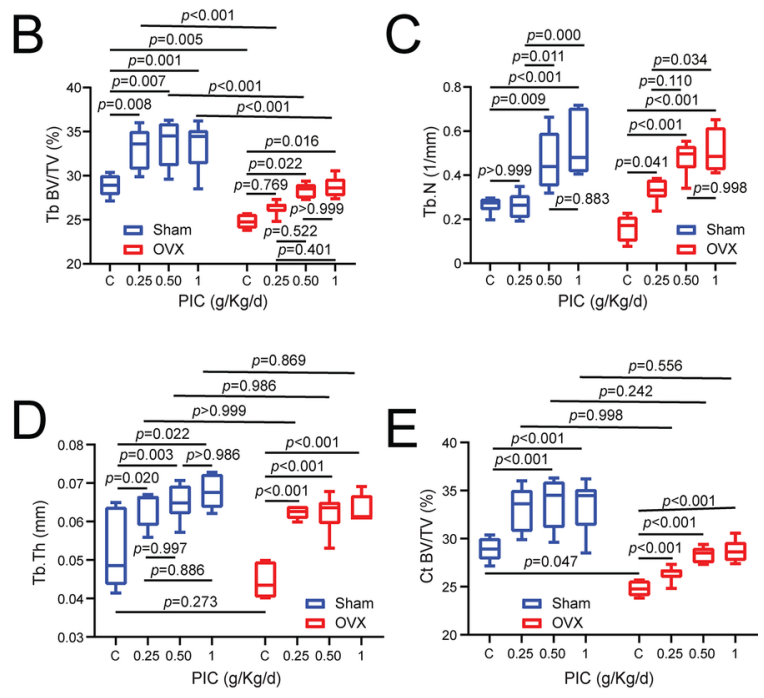
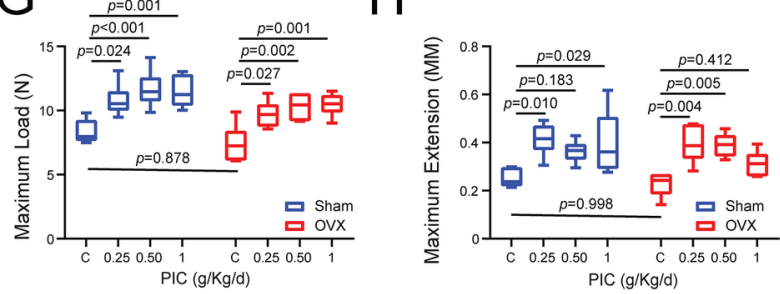

JBMR_4125_Figure 3.tif 
A
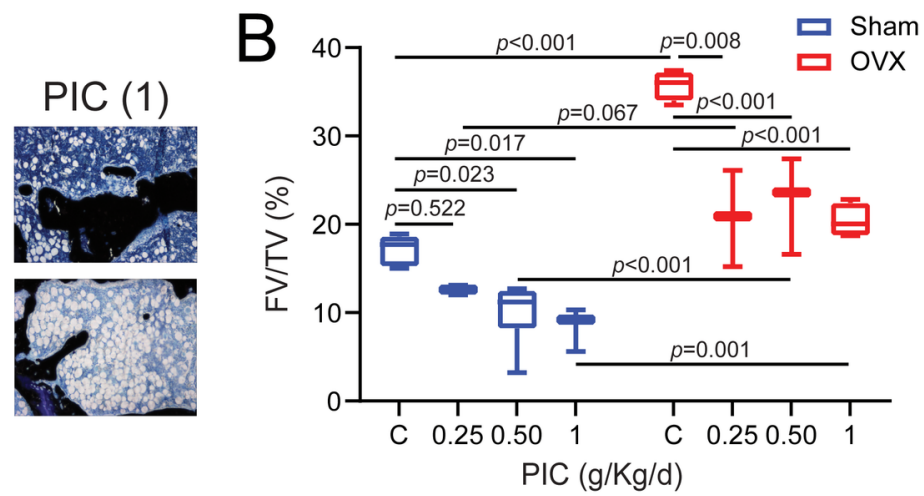

JBMR_4125_Figure 4.tif 
A

Sham
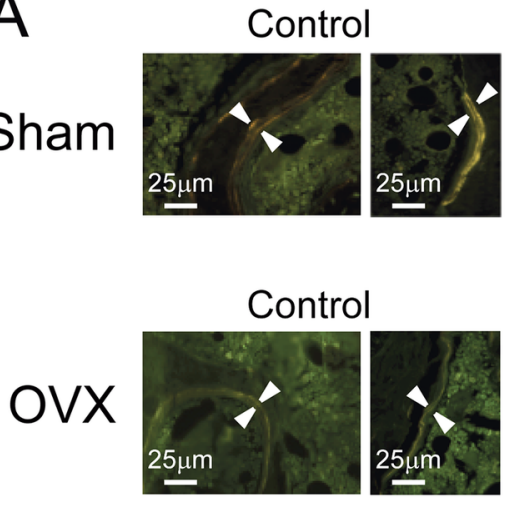

B

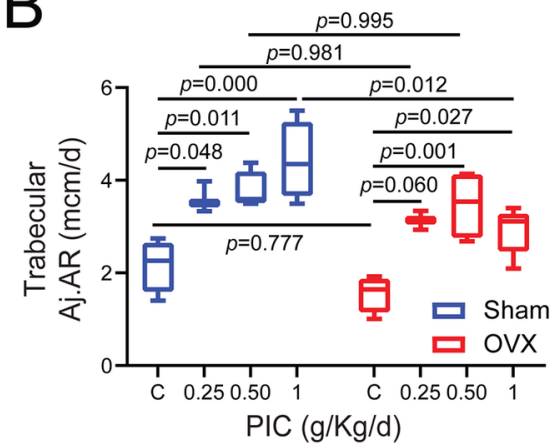

D

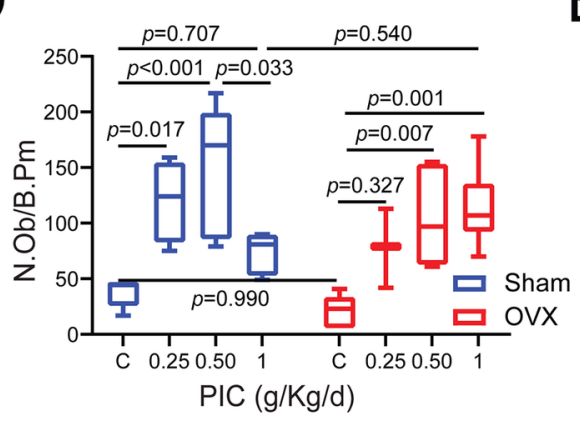

PIC (0.25)
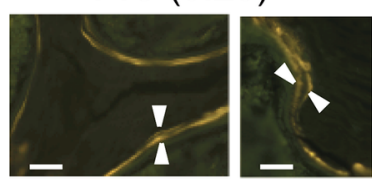

PIC (0.25)

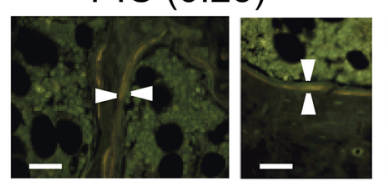

PIC (0.5)
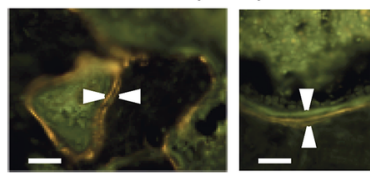

PIC (0.5)
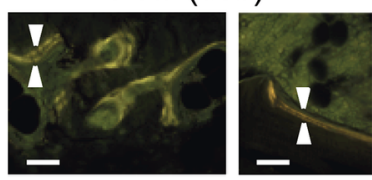

PIC (1)

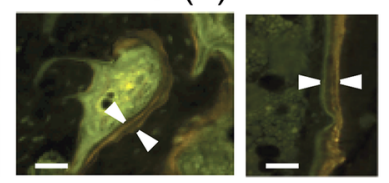

PIC (1)
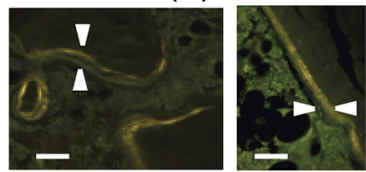

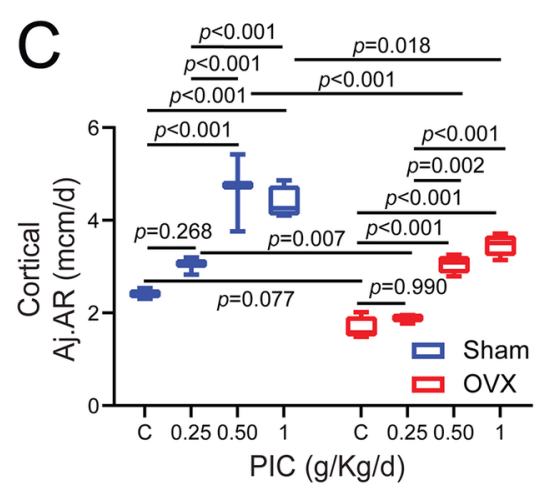

E

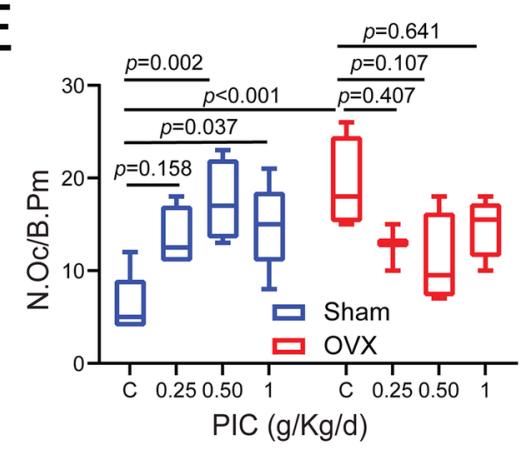

JBMR_4125_Figure 5.tif

This article is protected by copyright. All rights reserved. 
A

Sham

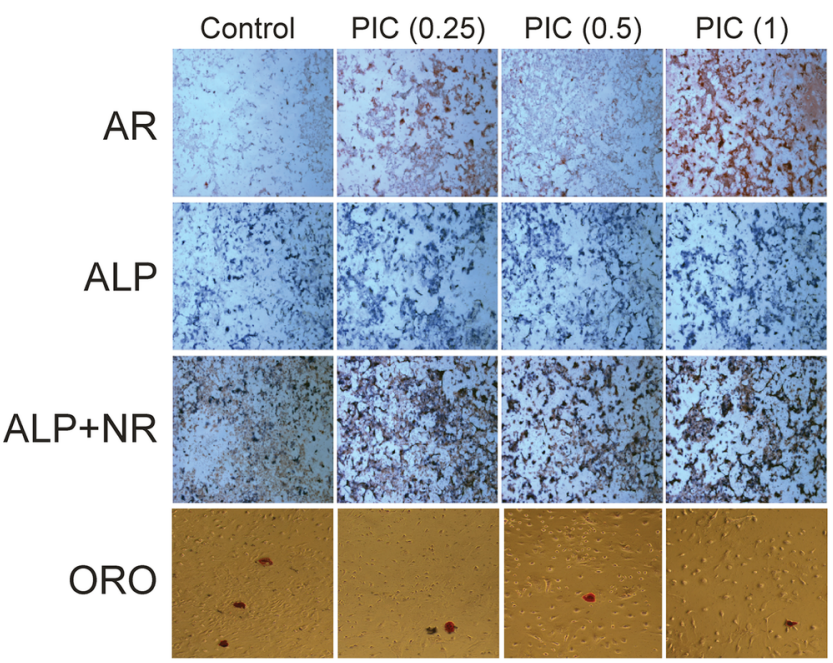

B

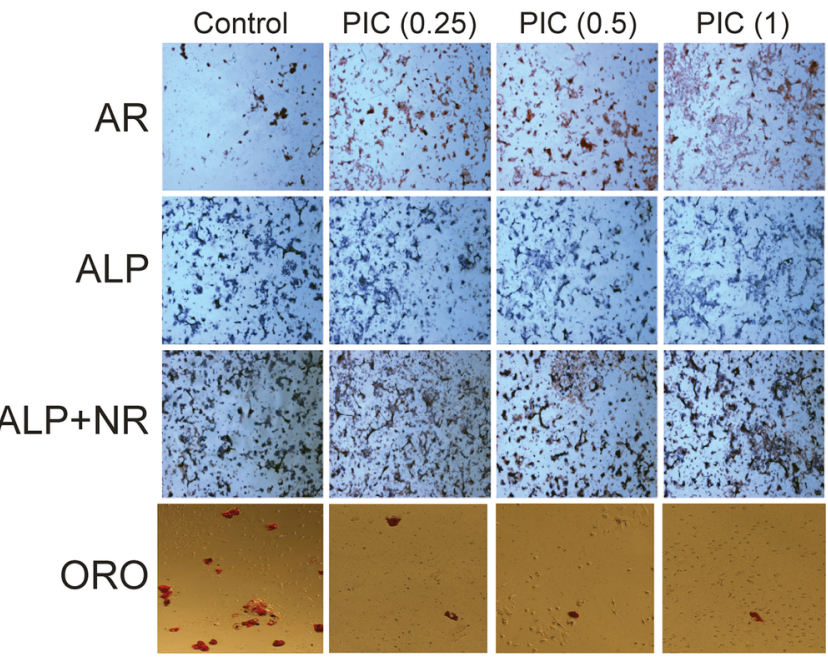

C

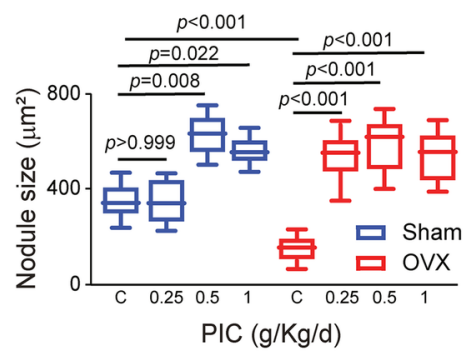

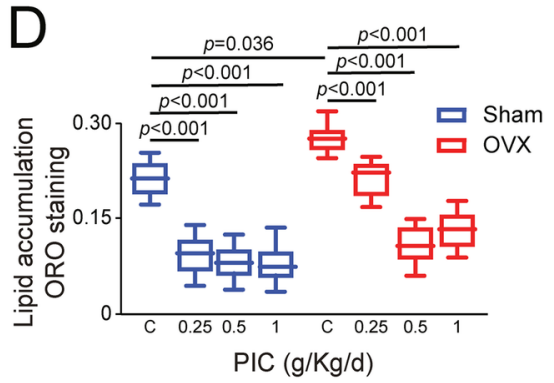

JBMR_4125_Figure 6.tif 

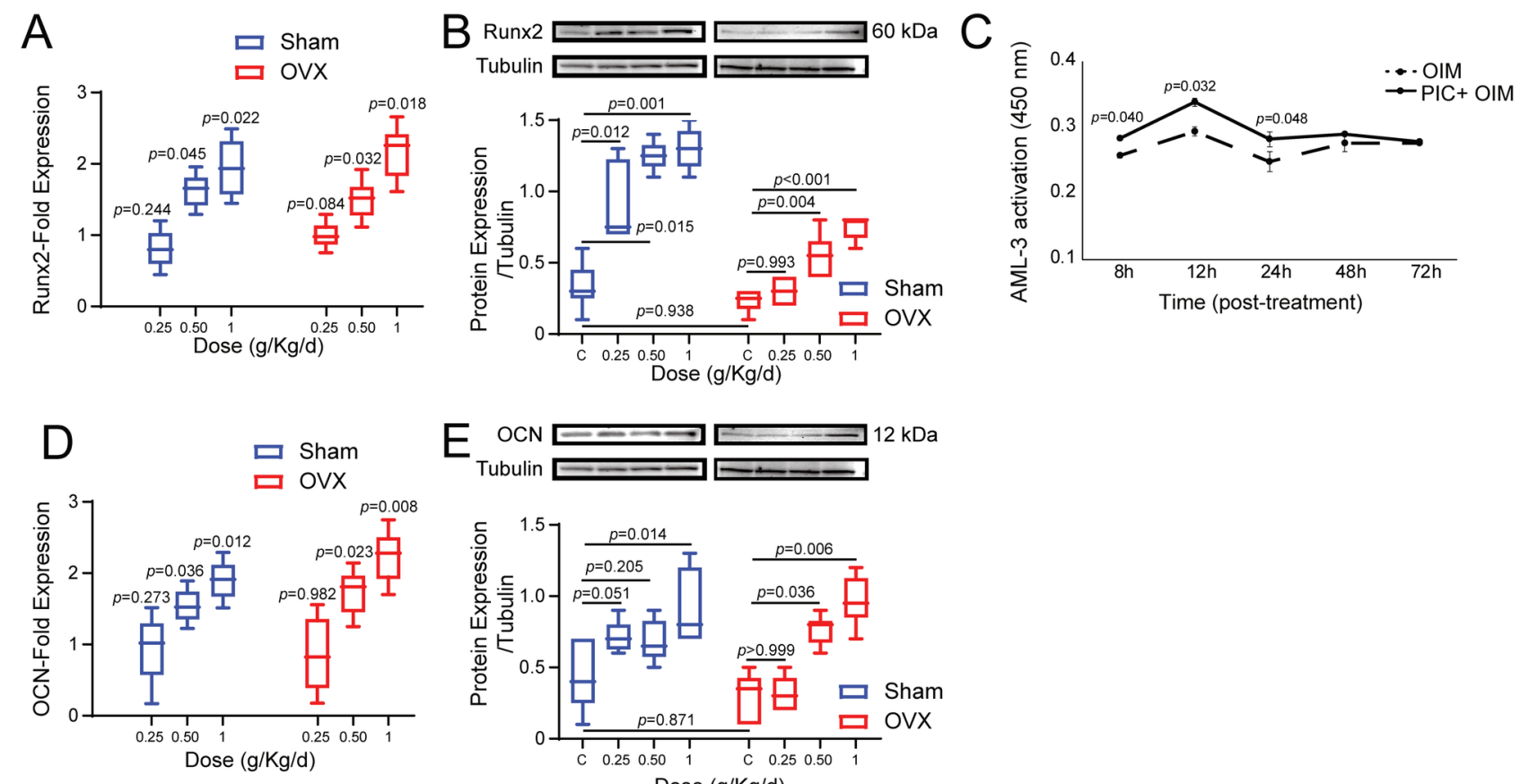

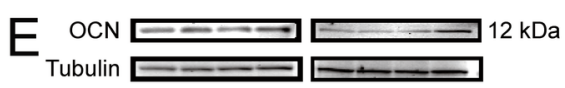

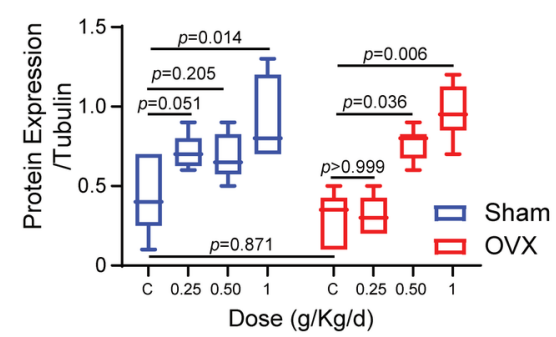

$\mathrm{F}$

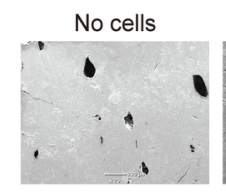

Control

G

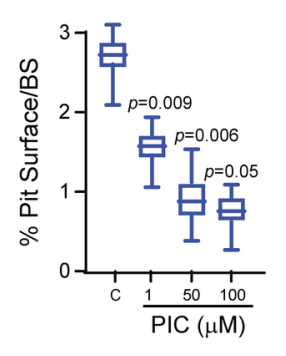

JBMR_4125_Figure 7.tif 


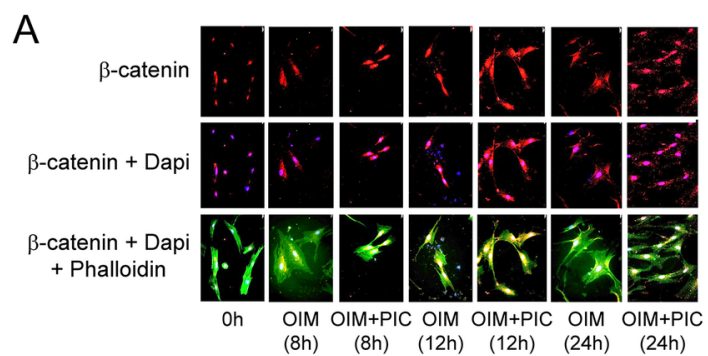

B
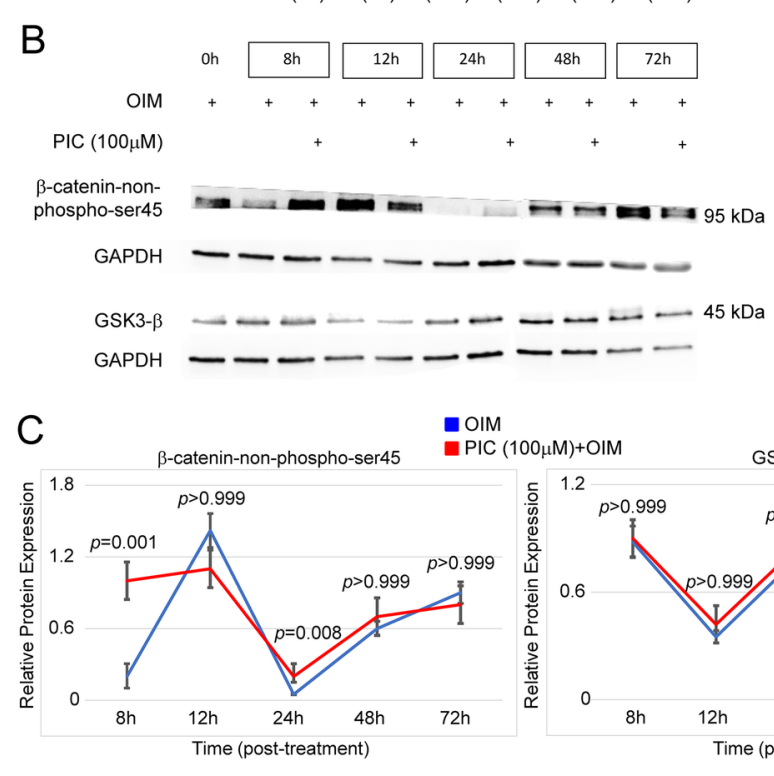

- OIM

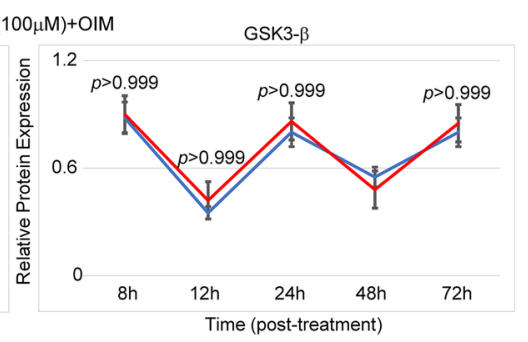

JBMR_4125_Figure 8.tif

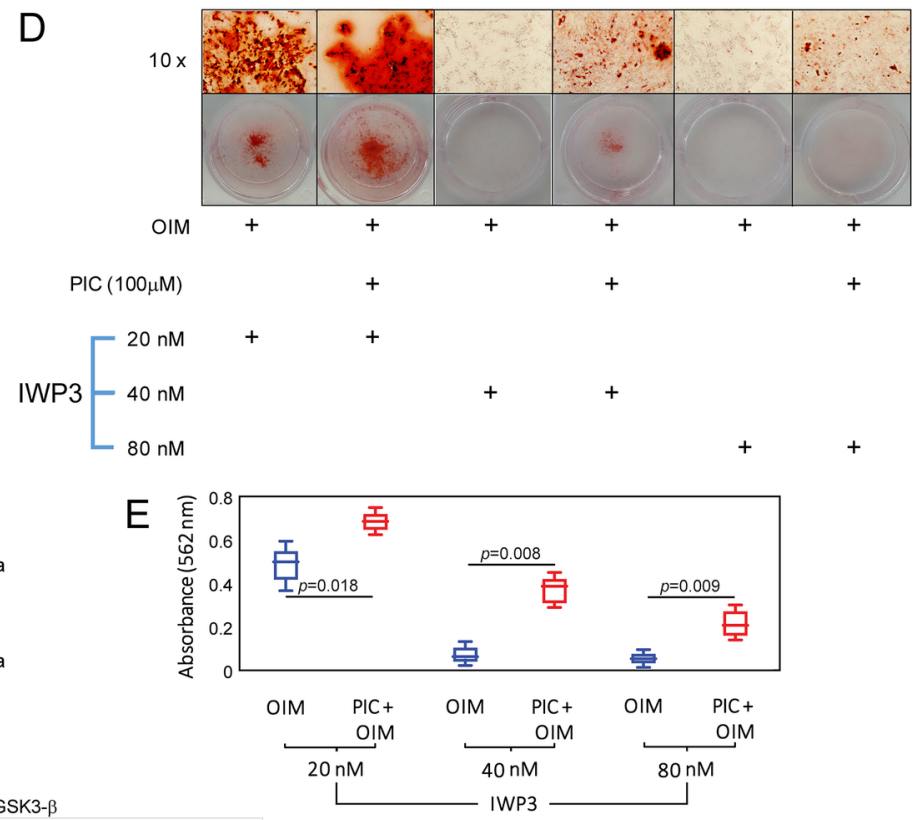




\section{University Library}

\section{- M M N E R VA A gateway to Melbourne's research publications}

Minerva Access is the Institutional Repository of The University of Melbourne

Author/s:

Duque, G;Vidal, C;Li, W;Al Saedi, A;Khalil, M;Lim, CK;Myers, DE;Guillemin, GJ

Title:

Picolinic Acid, a Catabolite of Tryptophan, Has an Anabolic Effect on Bone In Vivo

Date:

2020-08-17

Citation:

Duque, G., Vidal, C., Li, W., Al Saedi, A., Khalil, M., Lim, C. K., Myers, D. E. \& Guillemin, G. J. (2020). Picolinic Acid, a Catabolite of Tryptophan, Has an Anabolic Effect on Bone In Vivo. JOURNAL OF BONE AND MINERAL RESEARCH, 35 (11), pp.2275-2288. https:// doi.org/10.1002/jbmr.4125.

Persistent Link:

http://hdl.handle.net/11343/276153 